\title{
Détection et thérapeutique des troubles temporo-mandibulaires en orthodontie
}

\author{
Emmanuelle Bocquet $^{1 *}$, Alexis Moreau ${ }^{1}$, Michel Dangur $^{2}$, Chantal Dangur $^{2}$ \\ 1235 boulevard de la Liberté, 59000 Lille, France \\ 2 Faculté de Chirurgie Dentaire, 1 place de Verdun, 59000 Lille
}

MOTS CLÉS :

Dysfonction temporo-mandibulaire /

Anatomo-physiologie / Disque articulaire /

Orthodontie

KEYWORDS:

Temporo-mandibular malfunction /

Anatomy and physiology /

Articular disc /

Orthodontics
RÉSUMÉ - L'orthodontiste est confronté chaque jour à des troubles de l'occlusion, dont la prise en charge lui est habituelle. En revanche, la gestion de troubles temporomandibulaires est moins évidente, que ce soit au niveau détection, diagnostic ou prise en charge thérapeutique. Aussi, une connaissance approfondie de l'anatomophysiologie de l'articulation temporo-mandibulaire, de ses mécanismes d'action est indispensable pour permettre au praticien de détecter et diagnostiquer les différentes pathologies résultant de phénomènes étiologiques variés. Le diagnostic rigoureux, au moyen d'un examen clinique approfondi et d'examens complémentaires axiographiques et radiologiques, du dysfonctionnement temporo-mandibulaire et de ses étiologies (notamment dento-alvéolaires et occlusales) permettra de mettre en place une thérapeutique appropriée, qu'elle soit orthodontique pure, ou en collaboration avec des confrères d'autres disciplines (occlusodontiste, chirurgien, ostéopathe...).

ABSTRACT - Diagnosis and treatment of temporo-mandibular disorders in orthodontics. Orthodontists are fully prepared to treat the problems of occlusion that they are called upon to deal with every day. On the other hand temporo-mandibular joint disorders present more obscure difficulties from the point of view of detection and diagnosis as well the management of their treatment. That is why a profound understanding of the anatomical and physiological functioning of the temporomandibular joint has become indispensable for today's orthodontists who are now asked to detect and diagnose an assortment of TMJ disturbances whose etiology may vary greatly. By performing a rigorous diagnostic procedure, based on a thorough clinical examination supported by careful axiographic and radiological studies, of temporo-mandibular malfunctioning and its underlying etiological causes, which are primarily dento-alveolar and occlusal in nature, orthodontists will be able to adopt an appropriate therapeutic approach that might be purely orthodontic or multidisciplinary and carried out with the collaboration of specialists in occlusion, oral surgery, and even osteopathy.

\section{Introduction}

Les dysfonctions cranio-mandibulaires suscitent des interrogations et des controverses quant à leur étiologie et leurs conséquences.

Pour Decker [17], «l'occlusion n'est plus considérée comme un facteur déterminant, mais reste un facteur aggravant».

De ce fait, l'orthodontie représente une solution thérapeutique majeure dans le traitement d'une

*Auteur pour correspondance : orthem@free.fr dysfonction de l'appareil articulaire. Ceci d'autant plus que certains troubles temporo-mandibulaires préexistent de manière plus ou moins silencieuse au traitement orthodontique, et qu'ils pourront être dévoilés par celui-ci.

\section{Notions d'anatomo-physiologie}

\subsection{Physiologie}

Lapparition de l'articulation temporo-mandibulaire traduit l'adaptation des mammifères aux 


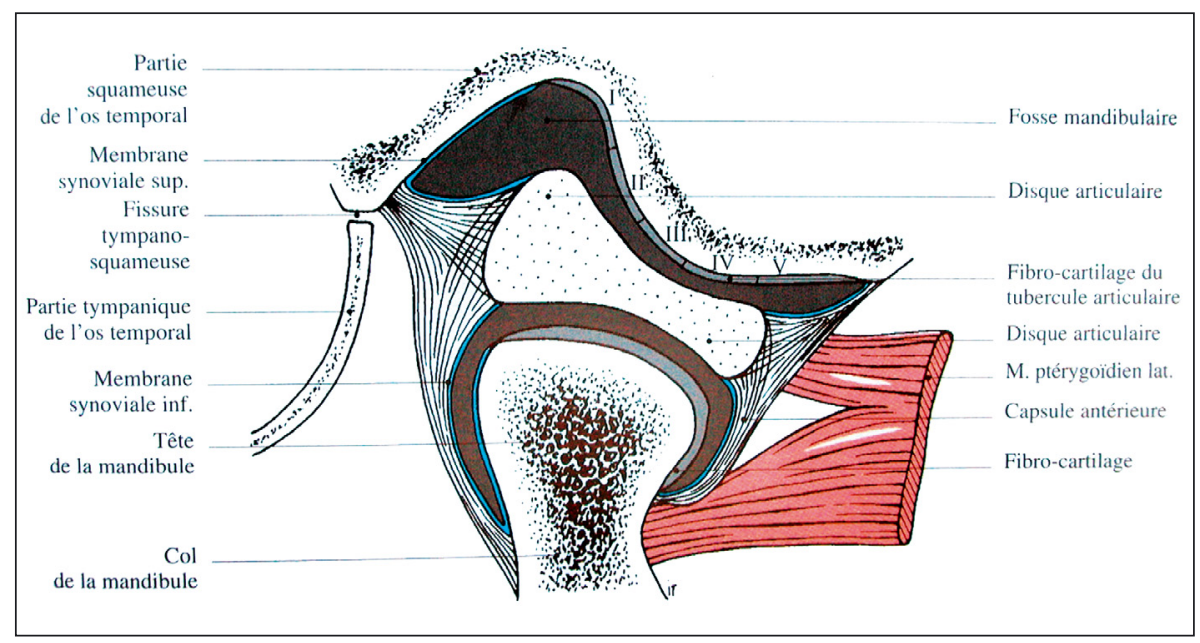

Figure 1

Coupe sagittale de l'articulation temporo-mandibulaire (d'après Fontaine, et al. [21]).

modifications morphologiques et fonctionnelles de leur squelette céphalique: posture érigée, mastication.

Les théories classiques actuelles mettent la plupart des transformations humaines sur le fait de la locomotion et du mode de vie [1, 10, 23].

Les articulations temporo-mandibulaires (ATM) opposent les condyles mandibulaires aux surfaces articulaires temporales et permettent tous les mouvements de la mandibule par rapport au maxillaire (ouverture, fermeture, propulsion, diduction).

Ces articulations sont des articulations synoviales bicondyliennes à fibrocartilage interposé.

\subsection{Anatomie}

\subsubsection{Les surfaces articulaires}

L'os temporal par l'intermédiaire du segment horizontal de la partie squameuse comprend la fosse mandibulaire en arrière et le tubercule articulaire en avant.

La fosse mandibulaire (cavité glénoïde), qui est une dépression concave vers le bas dont la base répond à la dépression qui sépare les tubercules zygomatiques, n'est pas recouverte de fibrocartilage.

Le tubercule articulaire (condyle temporal) correspond à la racine transverse du processus zygomatique et répond latéralement au tubercule zygomatique antérieur.

Du point de vue fonctionnel, on divise cette zone en trois segments (Fig. 1):

- le fond de la fosse mandibulaire en arrière : zone I de rétrocondylie,
- la pente condylienne divisée, d'arrière en avant, en zone II d'occlusion centrée, zone III de repos ou de relation centrée, zone IV de crête,

- le versant antérieur du tubercule articulaire : zone $\mathrm{V}$ en plateau.

Il est une notion anatomique essentielle à admettre : il n'existe pas de "ménisque » isolé (comme il en existe au niveau du genou) au sein de l'ATM, mais un disque.

Ce disque est le tendon du faisceau supérieur du muscle ptérygoïdien latéral, différencié en regard des surfaces articulaires. Il reçoit par ailleurs des insertions des muscles masséter profond et temporal postérieur : ces trois entités musculaires forment l'appareil tenseur du disque.

Ainsi est formée avec la tête condylienne une unité physiologique : le complexe condylo-discomusculaire [26] (Fig. 2).

\subsubsection{Les moyens d'union $[21,25,26]$}

La face profonde de la capsule articulaire prend attache au bord périphérique du disque articulaire et sépare ainsi l'articulation temporo-mandibulaire en un étage supérieur « disco-temporal » et un étage inférieur « disco-mandibulaire».

La capsule contient des fibres profondes temporo-discales dont les postérieures plus épaisses forment le frein discal postérieur (ligament de rappel de Poirier) riche en fibres élastiques et en récepteurs nerveux : ce ligament évite la luxation de la mandibule et du disque en avant du tubercule 


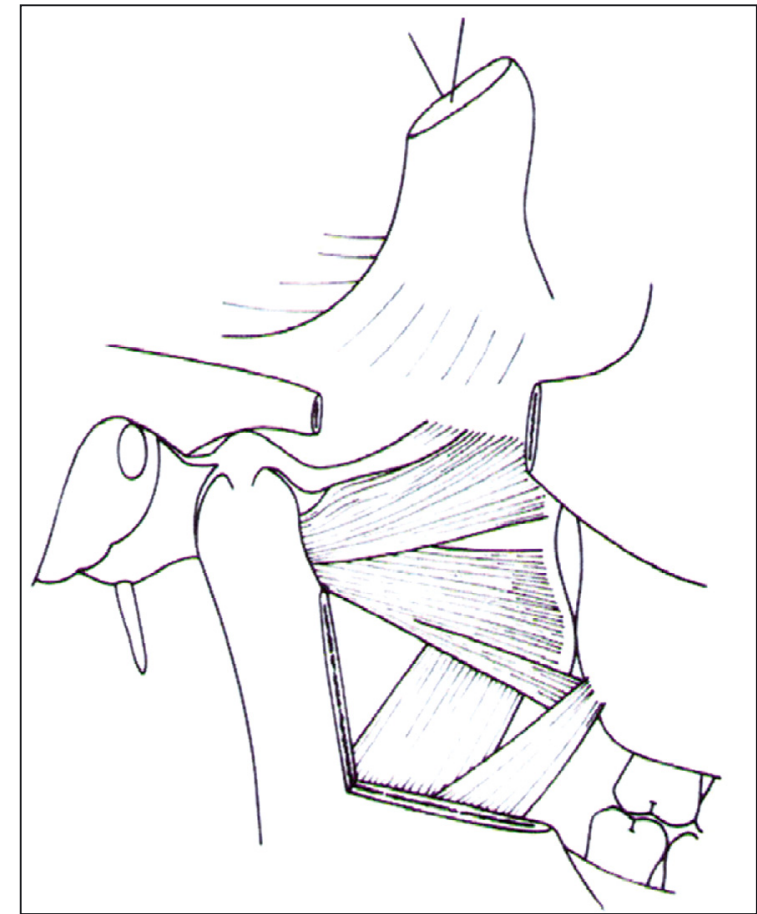

Figure 2

Complexe condylo-disco-mandibulaire (d'après Gola, et al. [25]).

articulaire temporal par un effet de rappel rétropulsif méniscal, et forme avec les fibres disco-temporales la zone bilaminaire des cliniciens.

Parmi les ligaments capsulaires (intrinsèques) renforçant la capsule sont présents :

- Le ligament latéral, très puissant, qui forme la corde zygomatico-maxillaire de Faraboeuf; il joue un rôle très important dans l'obtention de la relation centrée (qui est une relation ligamentaire cranio-mandibulaire) et lors des mouvements de diduction, afin de prévenir tout recul intempestif. Toute pathologie articulaire commence par l'élongation de ce ligament inextensible.

- Le ligament médial, qui est très lâche et joue un rôle accessoire.

Les ligaments extra-capsulaires (extrinsèques) sont des épaississements du fascia interptérygoïdien :

- Le ligament sphéno-mandibulaire joue un rôle important dans le concept ostéopathique puisqu'il met en relation le sphénoïde et la mandibule au niveau de l'Épine de Spix. Cette insertion est en fait, pour Delaire, le principal moteur de la croissance postérieure du corps mandibulaire. De plus, cette insertion voisine du centre de rotation de l'articulation temporo-mandibulaire évite que le paquet vasculo-nerveux alvéolaire inférieur soit étiré au cours des mouvements de la mandibule.

- Le ligament ptérygo-mandibulaire considéré comme l'insertion tendineuse des muscles constricteurs supérieurs du pharynx.

Il existe également deux ligaments otomandibulaires reliant l'ATM à l'oreille moyenne, vestiges de la phylogenèse de l'articulation quadrato-articulaire des reptiles vers l'ATM des mammaliens $[9,26]$.

- Le ligament disco-malléaire, fibreux et inextensible, correspond à l'insertion primitive du muscle ptérygoïdien latéral au niveau de l'extrémité postérieure du cartilage de Meckel. Il limite les excursions discales antérieures; sans être responsable des manifestations otologiques (acouphènes) des troubles temporo-mandibulaires, qui sont issues du spasme du muscle tenseur du tympan.

Il correspond au frein méniscal postérieur de Sappey.

- Le ligament malléo-mandibulaire, involution fibreuse du cartilage de Meckel, peut subir des tractions excessives lors d'ouverture buccale forcée, de chirurgie articulaire, de désarticulation temporomandibulaire et être ainsi à l'origine de luxations des osselets.

Le déplacement médial du disque au contact de ce ligament peut entraîner des paresthésies linguales.

- Le ligament de PINTO reliant le muscle sternocléido-mastoïdien à l'angle mandibulaire correspond à la terminaison conjointe des ligaments discomalléaire et malléo-mandibulaire.

\subsubsection{Les muscles moteurs $[21,26,30]$}

(Fig. 3, 4 et 5)

Le muscle masséter est formé d'une partie superficielle, d'une partie profonde et d'une partie moyenne (faisceau de Winslow).

Le masséter superficiel est propulseur et élévateur de la mandibule.

Le masséter moyen joue le rôle de régulateur du glissement antéro-postérieur.

Le masséter profond limite l'extension du mouvement condylien antérieur et ainsi limite l'ouverture maximale de la bouche. 


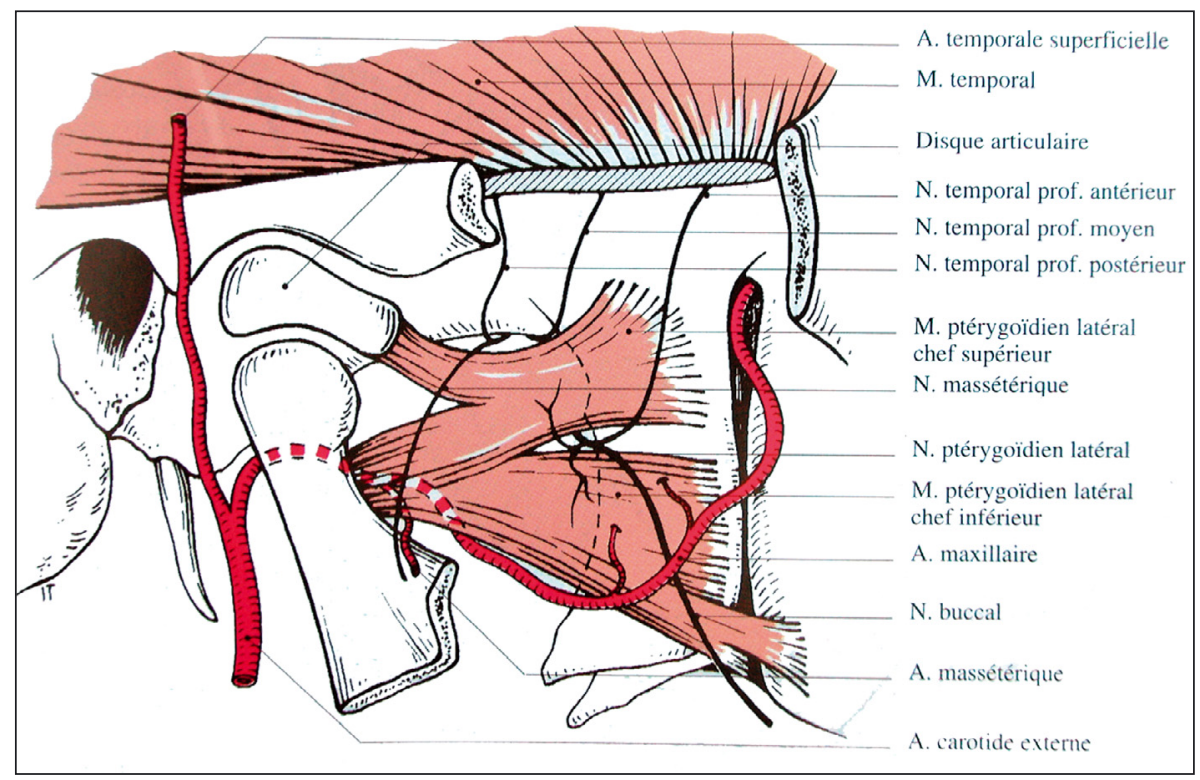

Figure 3

Vue latérale de la fosse infra-temporale (d'après Fontaine, et al. [21]).

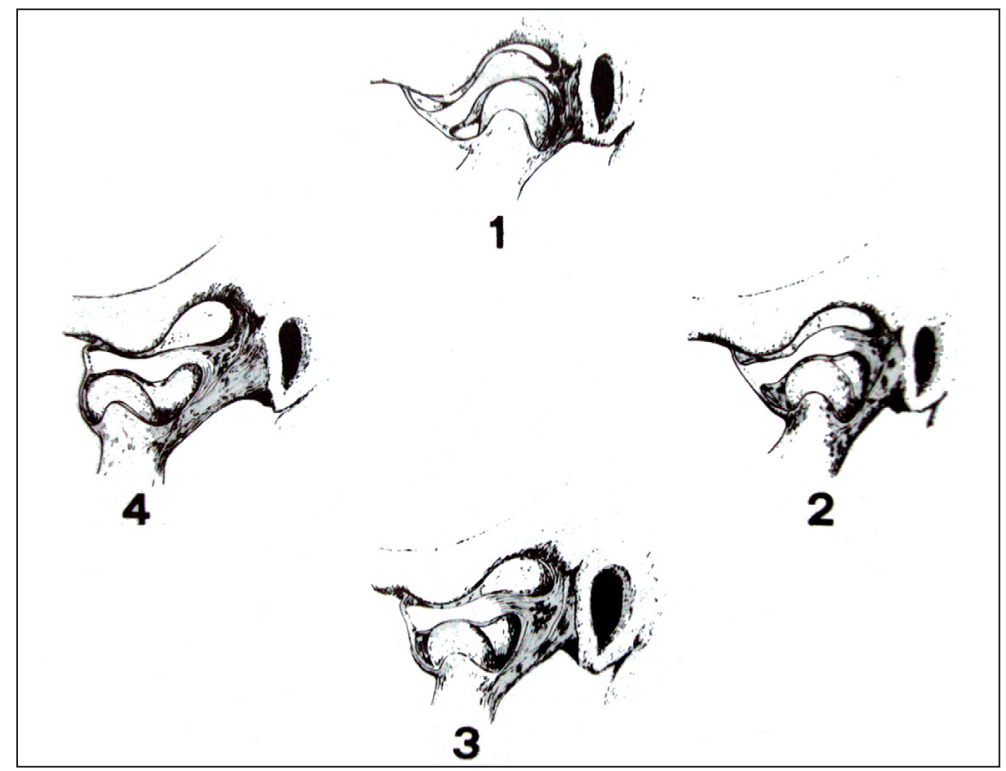

Figure 4

Rapports normaux entre le ménisque et la tête condylienne en position d'intercuspidation maximale, et pendant le mouvement d'ouverture (d'après Wilkes en Mongini, et al. [32]).

Le muscle temporal occupe toute la fosse temporale où sa contraction est visible et palpable selon l'orientation de ses fibres antérieures, moyennes et postérieures.

Le temporal antérieur est actif lors de la fermeture et régule le glissement antérieur.

Le temporal moyen agit lors de la rétrusion et de la fermeture et régule la dimension verticale d'occlusion.
Le temporal postérieur est actif en rétrusion et diduction.

Le muscle ptérygoïdien latéral prend une insertion sur le disque articulaire et sur le condyle par l'intermédiaire d'un faisceau supérieur et d'un faisceau inférieur.

Le faisceau supérieur joue un rôle dans l'élévation et la rétrusion mandibulaire, et stabilise la situation antérieure du complexe disco-condylien. De 


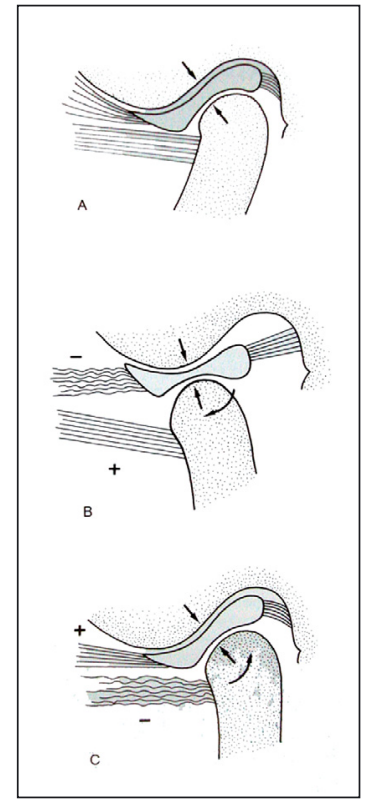

Figure 5

Action coordonnée des diverses structures anatomiques lors des déplacements condyliens. (A) Au repos. (B) Au cours de la propulsion, le chef inférieur du muscle ptérygoïdien latéral est activé. (C) Au cours de la rétropulsion, le chef supérieur du muscle ptérygoïdien est activé (d'après Mahan en Mongini, et al. [32]).

plus, un mélange de ses fibres avec le muscle orbitalis par la fissure orbitaire explique les relations qui existent entre l'ATM et l'occulo-motricité.

Le faisceau inférieur a un rôle propulseur, abaisseur et diductif du côté non travaillant en contraction unilatérale. Ce faisceau inférieur est en rapport avec le foramen ovale (V3) et l'artère méningée moyenne, ce qui expliquerait l'hypothèse étiologique des algies neuro-vasculaires de la face (Fig. 6).

Le muscle ptérygoïdien médial forme avec le muscle masséter un hamac musculaire autour de l'angle de la mandibule. Il a un rôle élévateur en contraction bilatérale et diducteur en contraction unilatérale.

Ainsi les principaux muscles masséter, temporal et ptérygoïdien latéral agissent à la fermeture et tirent le ménisque en avant et en dedans. Caix [7] parle de complexe condylo-ménisco-musculaire.

Lappareil moteur du disque articulaire existe et se contracte à la fermeture en synergie avec les muscles élévateurs de la mandibule. Cet appareil contrôle ainsi le devenir de la position condylienne instable qui se déplace crânialement de façon active par la contraction des élévateurs pour la

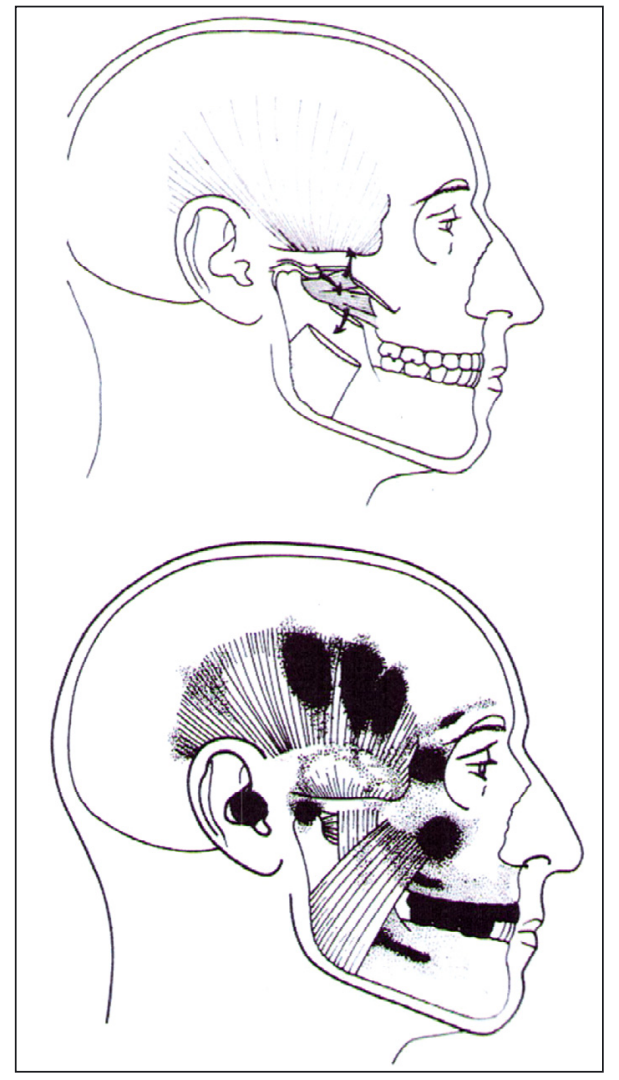

Figure 6

Extension du spasme du ptérygoïdien latéral aux autres muscles manducateurs (d'après Gola, et al. [25]).

propulsion-diduction, et caudalement de façon passive par la relaxation contrôlée des élévateurs et la rétraction élastique du ligament de Poirier.

Lappareil moteur du disque articulaire a pour fonction essentielle le maintien d'une posture discale antérieure et le contrôle de la vitesse de rétropulsion disco-condylienne.

Le système est donc passif à l'ouverture et actif à la fermeture : il s'agit du concept de Carpentier et Yung [30].

\subsubsection{La vascularisation}

De nombreuses artères contribuent à la vascularisation, par l'intermédiaire de leurs troncs ou de leurs branches:

- l'artère temporale superficielle,

- l'artère temporale profonde moyenne,

- l'artère maxillaire,

- les artères auriculaires postérieures,

- les artères pharyngiennes ascendantes. 
Les veines ont tendance à former un manchon veineux autour du condyle; elles se drainent vers le plexus parotidien et le système jugulaire externe.

Quant au réseau lymphatique, il se déverse dans les ganglions parotidiens et prétragiens.

\subsubsection{Linnervation [21]}

Le nerf trijumeau, $\mathrm{V}^{\mathrm{e}}$ nerf crânien originaire du premier arc branchial, est un nerf mixte à vocation sensitive et motrice :

- La branche sensitive renferme l'important ganglion de Gasser et se distribue ensuite en nerf ophtalmique, nerf maxillaire et nerf mandibulaire.

- La branche motrice est dédiée au nerf mandibulaire. Elle innerve les muscles masticateurs.

- Une fonction viscéro-motrice sécrétoire est assurée par des fibres du trijumeau dont les corps cellulaires sont situés dans les noyaux des $\mathrm{VII}^{\mathrm{e}}$ et IX ${ }^{e}$ nerfs crâniens (noyau lacrymal du nerf facial, noyau salivaire crânien du nerf intermédiaire, noyau salivaire caudal du nerf glossopharyngien).

Les articulations temporo-mandibulaires siamoises et couplées entre elles sont gérées par un système neurologique performant d'un haut niveau d'intégration qu'est le système trigéminal.

Leur innervation est assurée par le nerf mandibulaire, par ses collatérales que sont :

- le nerf auriculo-temporal pour la région postérieure et latérale de la capsule, ainsi que pour la zone bilaminaire,

- les nerfs temporaux profonds postérieur et massétérin pour la partie antérieure de la capsule,

- le nerf lingual qui innerve le ligament ptérygomandibulaire,

- par les rapports étroits qu'entretient le nerf mandibulaire avec le fascia interptérygoïdien, le ligament sphéno-mandibulaire et le ligament latéral interne au niveau de la scissure de Glaser.

Ainsi, la mandibule est reliée au crâne, au sphénoïde et au temporal par un système musculo-ligamentaire innervé par le trijumeau. Le système cranio-sacrémandibulaire est sous la dépendance du V (Fig. 3).

\subsection{Le rôle de l'ATM $[10,21]$}

On considère que la mastication est la fonction principale de l'appareil manducateur bien que la conception de l'ATM ne la prédestine pas au travail (faible force de résistance du disque, structure fibro-cartilagineuse du condyle qui est plus apte à des mouvements d'étirement que de compression).

Mais le temps dédié à la mastication, estimé par certains auteurs à 16 minutes par jour, semble diminué par une augmentation de l'ingestion d'aliments mous, et par une diminution des temps de repas.

Or un manque de stimulation de la chaine masticatrice entraîne des problèmes physiologiques par manque de stimulation des muscles masticateurs et de l'ATM (puisque la croissance du cartilage condylien serait de nature adaptative sous influence des muscles), mais aussi des problèmes psychologiques, puisque cette chaine masticatrice est celle de la vie de relation et de l'extériorisation.

\section{Formes cliniques et notions générales de thérapeutique}

Le système dentaire, sensible à notre intervention, mais ne possédant pas de capacité réelle d'adaptation, doit être intégré par nos soins dans la physiologie générale de l'appareil manducateur, afin de ne pas devenir par son arrangement un élément pathogène pour tous les autres constituants auxquels il impose en fait sa loi.

\subsection{Les dysfonctions cranio-mandibulaires $[8,15,16,19,20,24]$}

Elles sont caractérisées par :

- une perte de l'intégrité du complexe discocondylien,

- un déplacement du disque en avant et en dedans,

- une perte partielle ou totale des attaches discales,

- une biomécanique altérée du disque,

- des troubles de la cinétique mandibulaire.

Une classification simplifiée définit la subluxation, la luxation réduite, la luxation non réduite aigue, la luxation non réduite chronique, la surrotation et l'ankylose.

\subsubsection{La subluxation (Fig. 7)}

C'est la pathologie la plus algique, mais de meilleur pronostic. Les signes constatés sont

- une inflammation de la zone bilaminaire postérieure appelée rétrodiscite, 


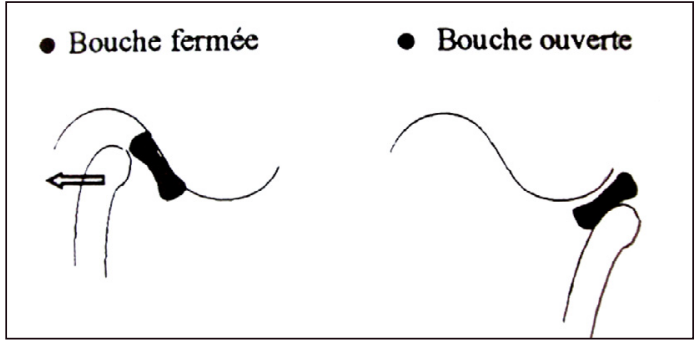

Figure 7

Schéma de subluxation (d'après Durand, et al. [20]).

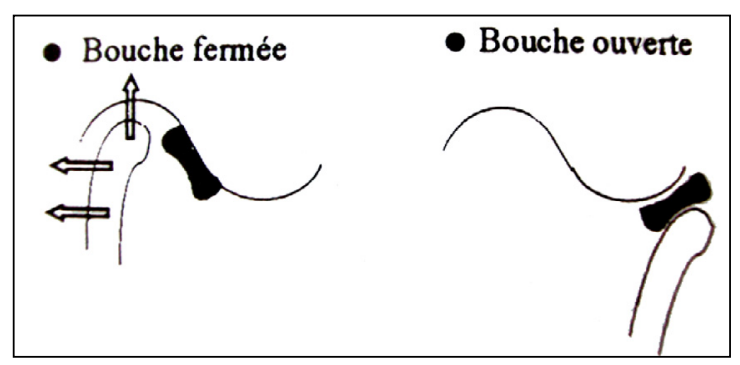

Figure 8

Schéma de luxation réduite (d'après Durand, et al. [20]).

- des myalgies,

- une absence de bruit articulaire,

- une cinétique mandibulaire normale,

- des otalgies,

- une palpation des ATM douloureuse en intraarticulaire,

- des attaches condylo-discales distendues mais conservées.

\subsubsection{La luxation réduite (Fig. 8 et 9)}

Les signes constatés sont :

- des algies,

- un bruit articulaire : un claquement en début d'ouverture quand le condyle capte le disque, puis un claquement en fin de fermeture quand le condyle perd le disque,

- une absence de limitation de l'ouverture buccale,

- une cinétique mandibulaire altérée avec ressaut, déviation lors du passage du bourrelet postérieur du disque,

- une perte des attaches uni- ou bipolaires avec un déplacement discal plus ou moins important,

- une biomécanique du disque altérée dans la phase primaire du disque comme expliqué ci-dessus.

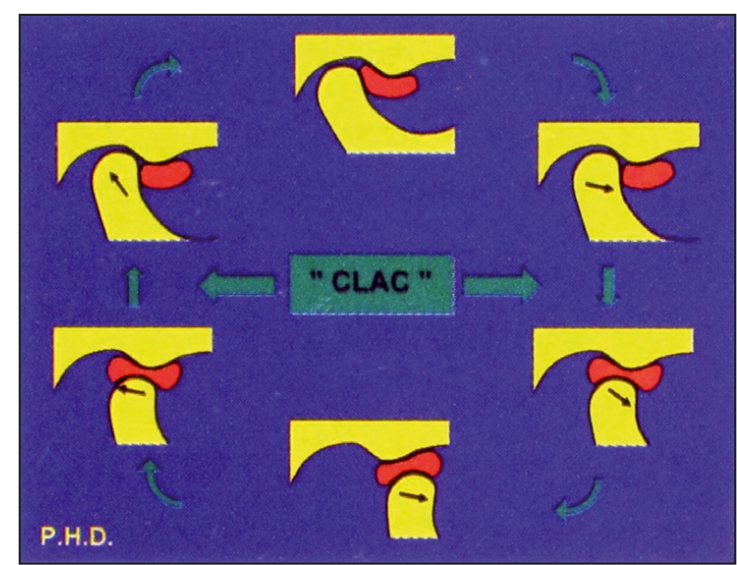

Figure 9

Antéposition méniscale réductible (d’après Dupas [19]).

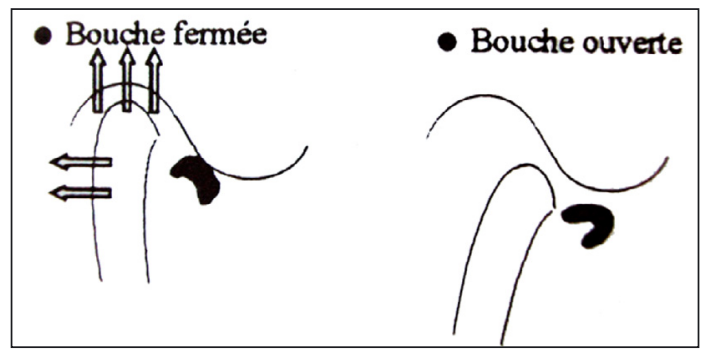

Figure 10

Schéma de luxation non réduite aigue (d'après Durand, et al. [20]).

\subsubsection{La luxation non réduite aiguë (Fig. 10)}

Les signes constatés sont :

- des algies importantes avec un sentiment d'angoisse face à des phases de blocage de la mandibule en ouverture,

- une limitation de l'ouverture buccale ne cédant pas à la contre-pression (diagnostic différentiel avec une atteinte musculaire) : il existe un fort abaissement condylien avec une importante contraction musculaire qui bloque l'ouverture buccale,

- une déflexion mandibulaire du côté atteint par abaissement condylien important sans coaptation du disque,

- une absence de bruit articulaire puisque le disque n'est pas coapté,

- une perte des attaches condylo-discales uni- ou bipolaires. 


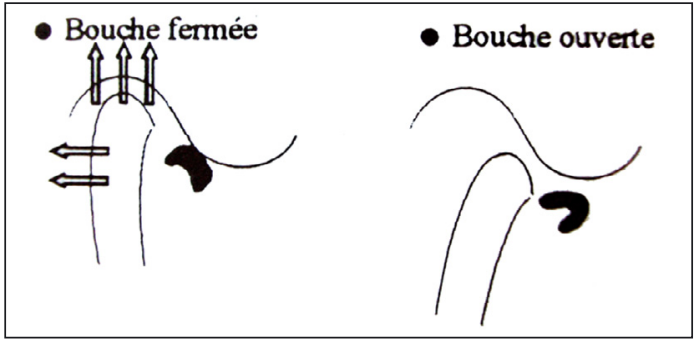

Figure 11

Schéma de luxation non réduite chronique (d'après Durand, et al. [20]).

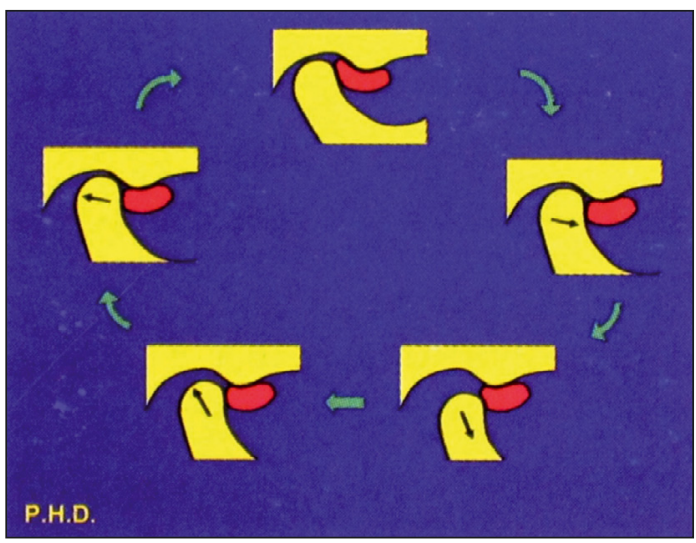

Figure 12

Antéposition méniscale irréductible (d'après Dupas [19]).

\subsubsection{La luxation non réduite chronique (Fig. 11 et 12)}

Les signes constatés sont :

- des algies variables avec une prépondérance des manifestations de la pathologie à distance,

- une amplitude des mouvements variable car il existe un étirement des tissus de soutien,

- un bruit articulaire de type crissement ou craquement,

- une perte totale des attaches condylo-discales,

- un déplacement très important du disque avec dégénérescence de celui-ci, ce qui explique le bruit articulaire,

- une déflexion du côté atteint. S'il n'existe pas trop de signes, il faut regarder la contraction des muscles sus- et sous-hyoïdiens en fin d'ouverture qui signe l'effort fait pour abaisser la mandibule.

\subsubsection{La surrotation}

Les signes constatés sont :

- un déplacement condylien antérieur important au-delà du bourrelet antérieur du disque,
- un claquement en fin d'ouverture expliqué par le passage du condyle en avant du disque,

- un risque de blocage en bouche ouverte quand le condyle ne repasse pas sous le disque en début de fermeture.

C'est une pathologie de fin d'ouverture qui peut, à force d'étirement ligamentaire, s'accompagner d'une luxation réduite.

\subsubsection{L'ankylose}

C'est le stade ultime avec un remaniement osseux ou fibreux, qui s'accompagne de mouvement très réduit voir inexistant avec des bruits articulaires importants.

\subsection{Etiologies et diagnostic différentiel [2, 3, 10, 17,33] (Fig. 13)}

Les étiologies sont variées et peuvent remonter à de nombreuses années suite à des évènements qui semblent parfois anodins pour le patient. D'où l'importance d'un interrogatoire minutieux et précis [11].

Le diagnostic différentiel sera à faire avec :

- un problème hormonal qui provoque des céphalées,

- des troubles neurologiques : névralgies, migraines,

- des troubles vasculaires : algies de la face,

- des pathologies ORL : sinusite, otite, lithiase salivaire,

- des maladies générales: psoriasis, tuberculose, toutes les formes de rhumatisme, les maladies sexuellement transmissibles.

Il est reconnu que ces troubles se rencontrent chez l'homme et la femme, à tout âge, même si une prépondérance est notée chez la femme jeune (la femme a-t-elle une vie plus stressante, ou consulte-t-elle plus facilement que l'homme?...). $50 \%$ de la population serait touchée, mais une personne sur dix consulte, la souffrance étant le principal motif de consultation [25].

\subsection{Moyens de diagnostic $[15,16,20]$}

L'axiographie est un enregistrement graphique des mouvements mandibulaires au niveau condylien. C'est un examen non invasif à bonne valeur diagnostique qui permet l'examen des rapports occlusaux 


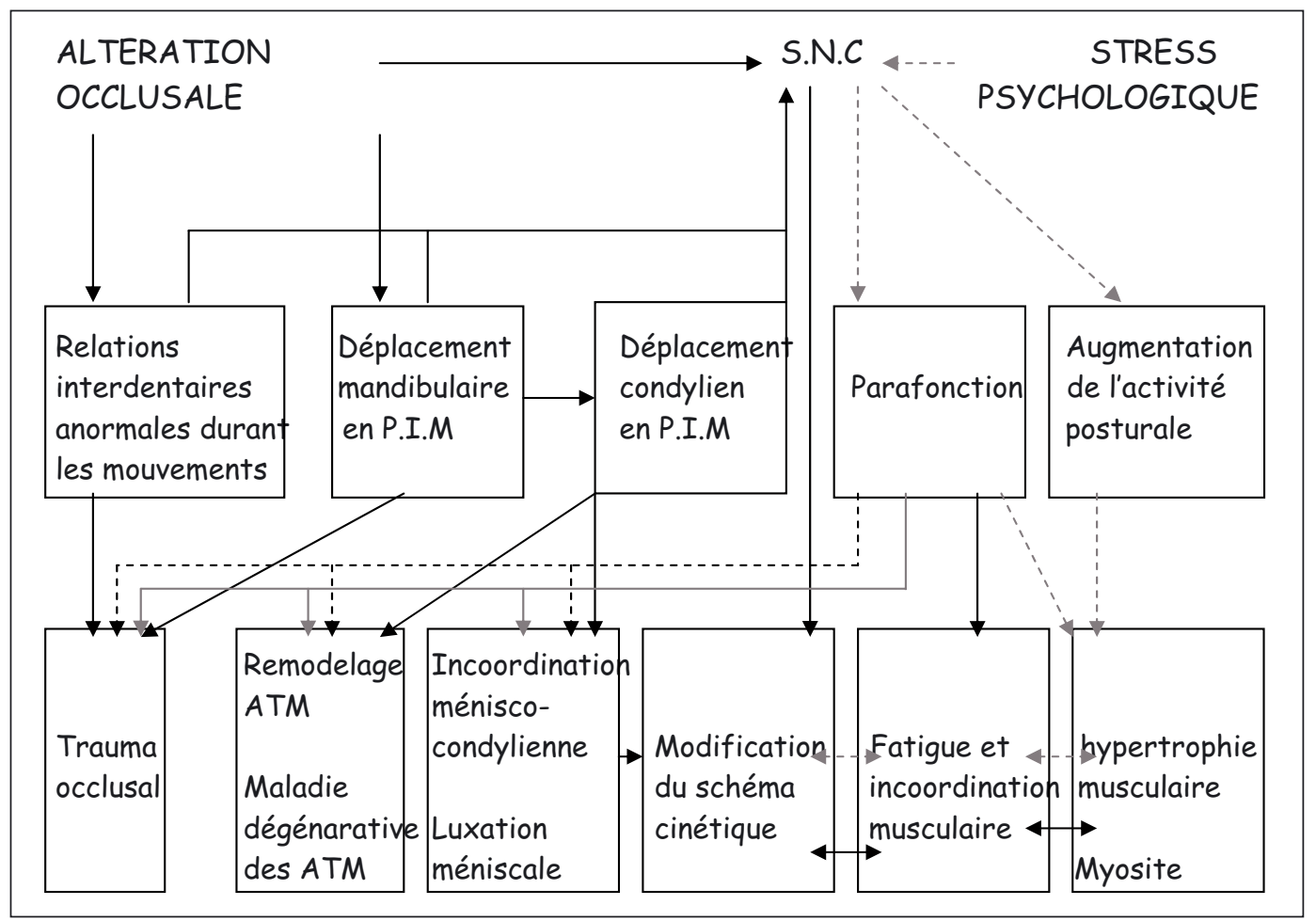

Figure 13

Les mécanismes de dysfonction du système stomatognathique. Les mécanismes qui sont sous la dépendance de facteurs psychologiques sont fléchés en gris, ceux qui sont sous la dépendance de facteurs occlusaux sont fléchés en noir (d'après Mongini, et al. [32]).

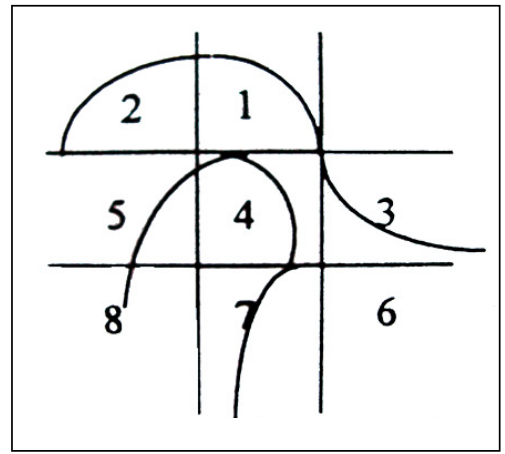

Figure 14

Position de Gelb (d'après Durand, et al. [20]).

entre la relation centrée et la position d'intercuspidation maximale. L'enregistrement permet ensuite la programmation de l'articulateur aux données anatomiques du patient.

Le choix de l'imagerie idéale sera fonction de la composante à explorer [4].

Les tomographies les plus usitées sont les hypocycloïdales en incidence de Hirtz (Fig. 14).

Lutilisation des IRM [22] se révèle être le moyen diagnostique complémentaire le plus fiable. Les IRM correspondent à l'image d'une tranche de l'objet reconstruite sur le mode matriciel à partir de profils atomiques enregistrés puis traités par ordinateur.

Une IRM de l'ATM permet de visualiser l'anatomie des tissus mous, la morphologie et la position du système disco-ligamentaire, la dynamique du complexe disco-condylien.

La normalité d'un cliché IRM correspond à un disque articulaire interposé entre le condyle et l'os temporal en bouche fermée et en bouche ouverte avec absence d'épanchement intra-articulaire.

Les figures 15 et 16 montrent quelques exemples de clichés IRM.

\section{Quelques solutions thérapeutiques [18, 20,36]}

Le traitement sera décidé après diagnostic, suite à :

- une demande du patient qui souffre d'une symptomatologie invalidante algique ou fonctionnelle locorégionale et/ou à distance, 


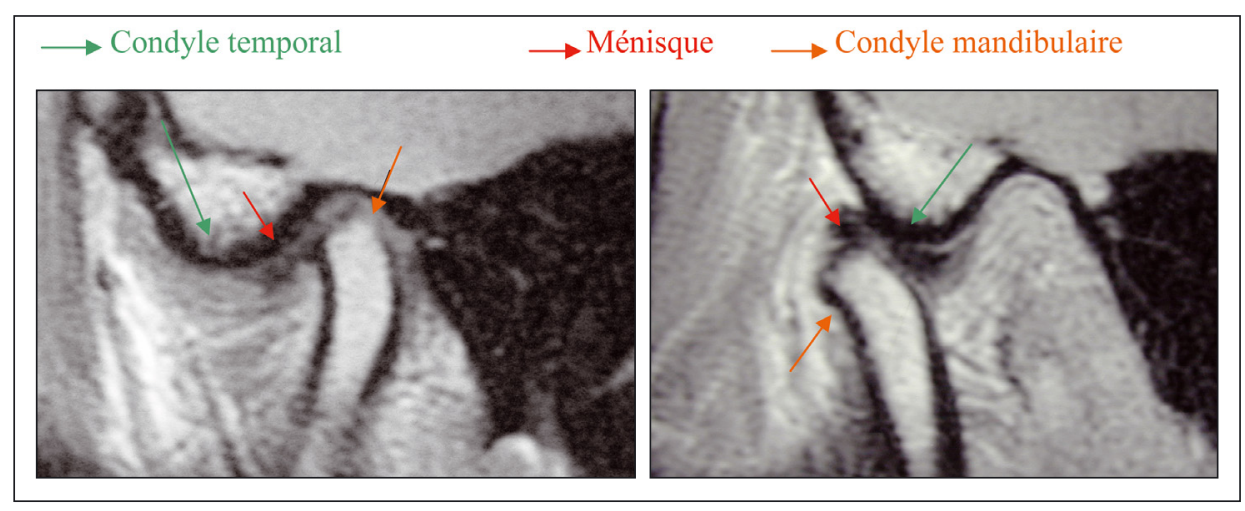

Figure 15

Clichés d'une ATM en subluxation (C Bocquet).

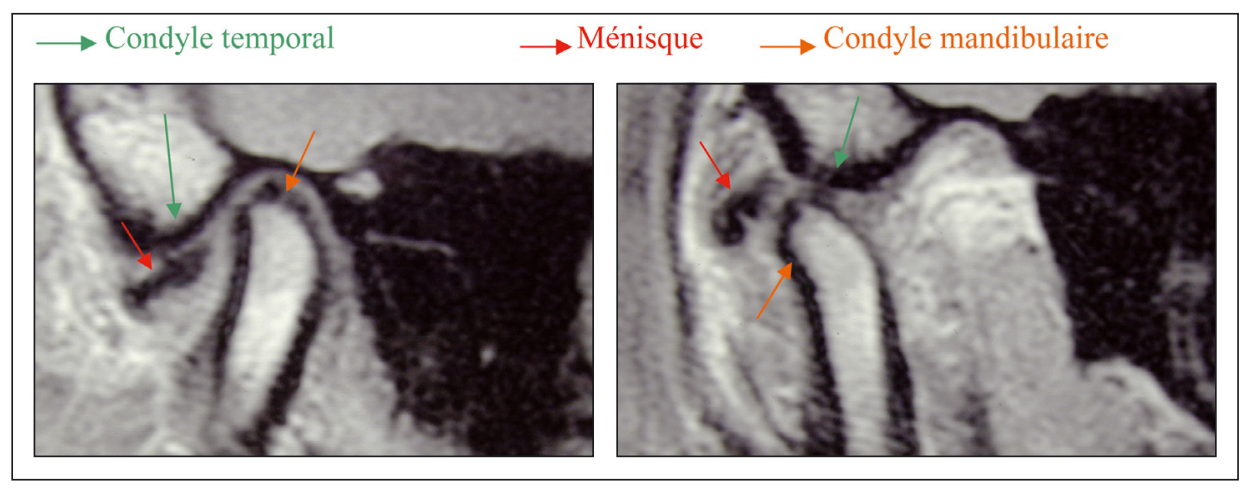

Figure 16

Clichés d'une ATM en luxation non réduite (C) Bocquet).

- une demande du praticien qui souhaite une position de référence mandibulaire (axe charnière fixe et reproductible) avant toute modification de l'équilibre acquis occlusalement; ou qui signale une perturbation du système postural, oculaire ou ORL dont l'étiologie est d'origine temporomandibulaire.

\subsection{Traitement articulaire}

Le but du traitement sera de :

- retrouver une articulation fonctionnelle asymptomatique par l'intermédiaire d'une thérapeutique articulaire qui permet de compenser la perte de substance,

- construire ensuite l'occlusion en harmonie avec les nouveaux déterminants articulaires obtenus par l'intermédiaire d'une thérapeutique de stabilisation.

Les objectifs temporo-mandibulaires seront d'obtenir un axe charnière de rotation de la mandibule fixe et reproductible, vrai (par récupération du disque) ou néoformé (par formation fibreuse qui remplace le disque), d'obtenir une absence de limitation des mouvements mandibulaires pour soulager le système postural $[31,32]$.

L'utilisation d'une gouttière rétablit l'équilibre neuro-musculaire, et par son action de repositionnement condylien orthopédique, permet la captation discale (dans $5 \%$ des cas) ou une densification fibreuse de la zone bilaminaire postérieure (Fig. 17).

Pour obtenir la densification de la zone bilaminaire postérieure, le condyle est abaissé de la valeur de l'épaisseur du disque, et ainsi le vide obtenu entraîne une formation fibreuse par les fibroblastes.

Mais cette compensation intra-articulaire entraîne de nouveaux composants dento-dentaires qu'il faut harmoniser par l'orthodontie ou la prothèse.

Une autre thérapeutique, devant être utilisée avec précaution et parcimonie, est la chirurgie discale [18]. Cette solution thérapeutique est peu usitée de par ses lésions iatrogènes (adhérences discales, fibrose, arthrose voire ankylose) et ses douleurs postopératoires [25]. 


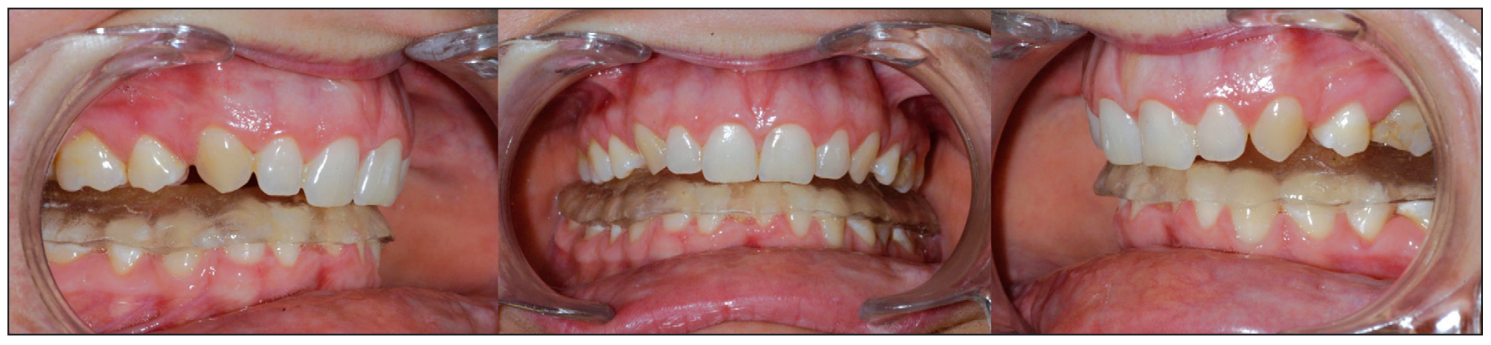

Figure 17

Gouttière de distension en bouche (C Bocquet).

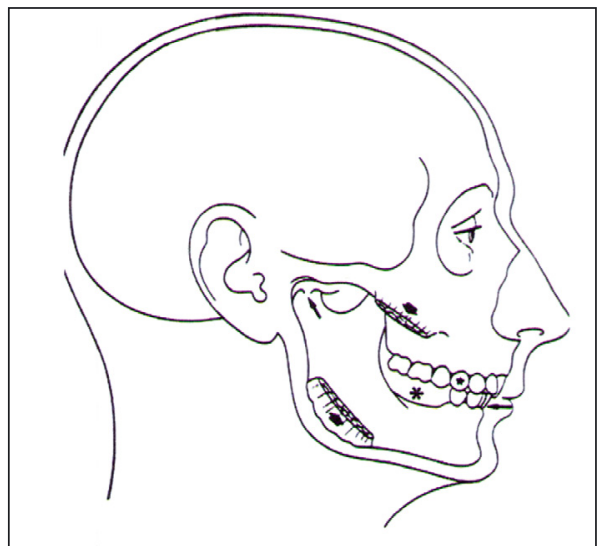

Figure 18

Facteurs de risques d'apparition d'un SADAM (d'après Gola, et al. [25]).

\subsection{Traitement orthodontique}

Certaines situations occlusales [27, 34, 35] sont des facteurs de risques d'apparition de troubles temporo-mandibulaires (Fig. 18), comme :

- un édentement non compensé (Fig. 19 et 20),

- une dysfonction du guide incisivo-canin par promandibulie/rétromandibulie compensée (version vestibulaire/palatine des incisives maxillaires) ou par afonction incisive (béance),
- une absence de liberté fonctionnelle par supraclusion incisive ou par articulé inversé latéral (Fig. 21).

\subsubsection{Dysfonction incisivo-canine}

Cette dysfonction peut être due à une béance incisive ou à une supraclusion majeure avec un articulé de type cléoaxial.

La thérapeutique corrigera dans un premier temps cette dysfonction avant de statuer sur la nécessité d'un traitement articulaire.

\subsubsection{Analyse architecturale}

Létude céphalométrique de Danguy [12-14] étudie la position du bord libre de l'incisive maxillaire par rapport à la bissectrice de l'angle Np-Es-Tm, points d'émergence du nerf trijumeau « grand architecte de la face " qui gère les proprioceptions linguales et labiales.

Ainsi, cette façon d'analyser la position incisive permet de poser précisément l'étiologie des dysmorphoses dento-alvéolaires et alors d'adapter la thérapeutique adéquate orthodontique, puis seulement articulaire si nécessaire (Fig. 22).

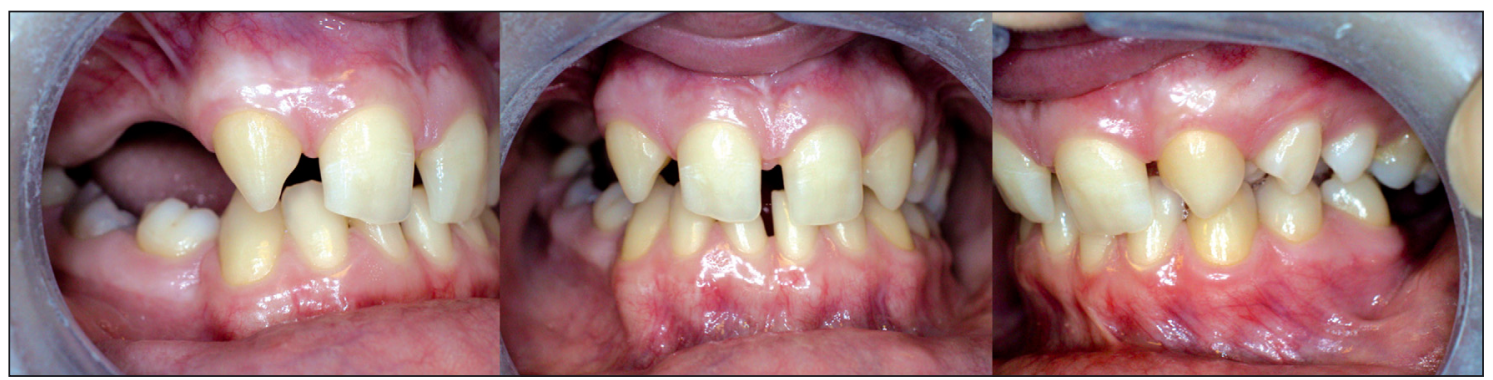

Figure 19

Cas de syndrome de Bloch-Sulzberger (incontinentia pigmenti) (C Bocquet). Au niveau buccal, les anomalies intéressent essentiellement les dents (90\% des cas) et les deux dentures, avec des retards d'éruption, des dysmorphies (dents coniques), une oligodontie, des diastèmes. 


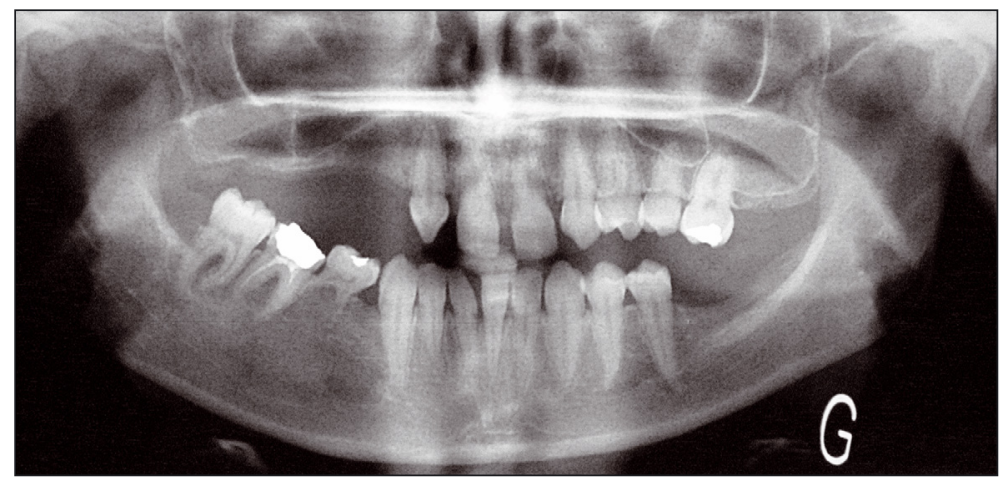

Figure 20

Panoramique du cas de Bloch-Sulzberger (c) Bocquet).

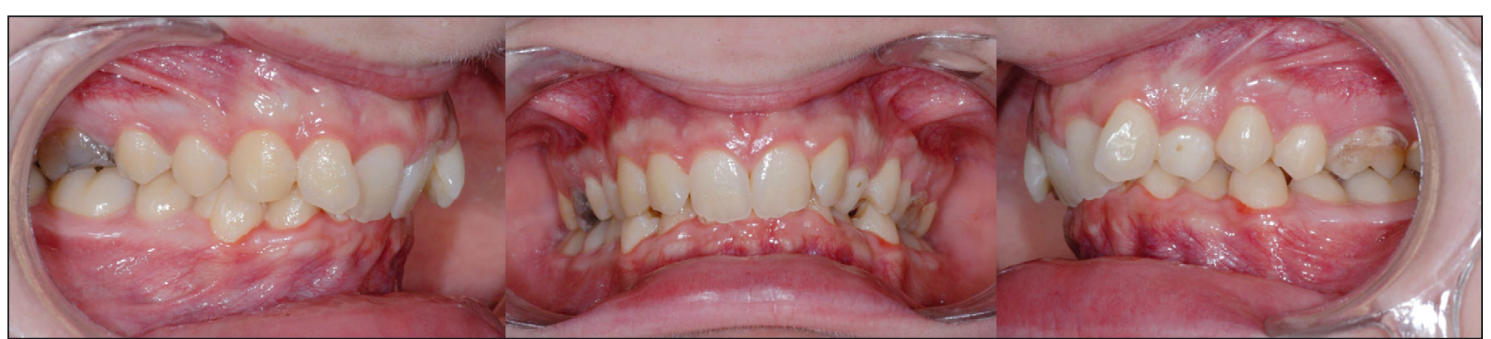

Figure 21

Cas d'absence de liberté fonctionnelle (C) Bocquet).

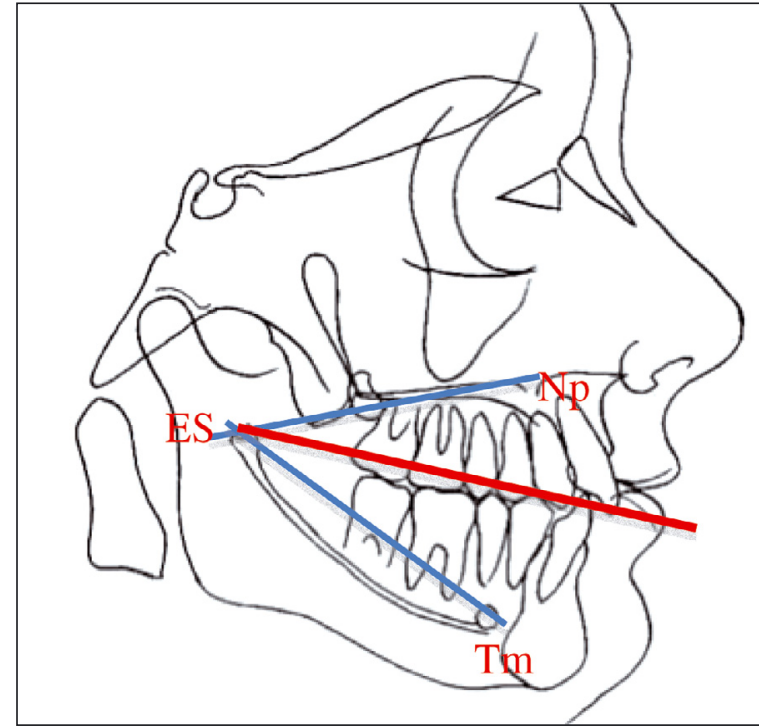

Figure 22

Analyse de la position verticale de l'incisive maxillaire (d'après Danguy [12-14]).

Le rebord incisif se trouve en position normale sur la bissectrice de l'angle Np-ES-Tm. Si l'incisive maxillaire coupe la bissectrice de plus de $1,5 \mathrm{~mm}$, il y a supra-alvéolie incisive maxillaire.

Danguy considère que d'une manière optimale, le rebord incisif mandibulaire doit se trouver verti-

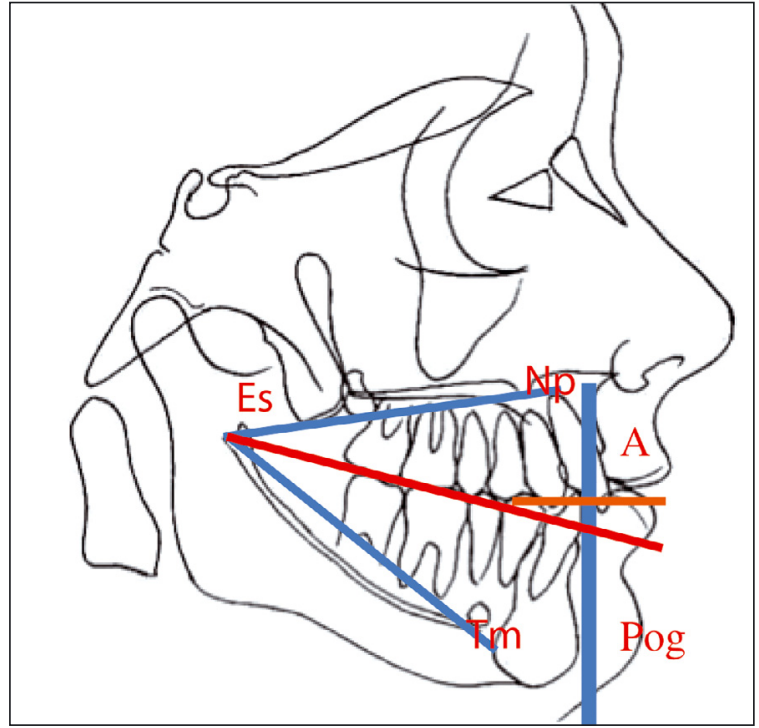

Figure 23

Analyse de la position verticale de l'incisive mandibulaire (d'après Danguy [12-14]).

calement sur la ligne APog et à $2 \mathrm{~mm}$ au dessus du point d'intersection de la bissectrice de l'angle NpEs-Tm et la ligne Apog, soit 2 à $3 \mathrm{~mm}$ au-dessus de la bissectrice en fonction de la dimension de l'incisive (Fig. 23). 


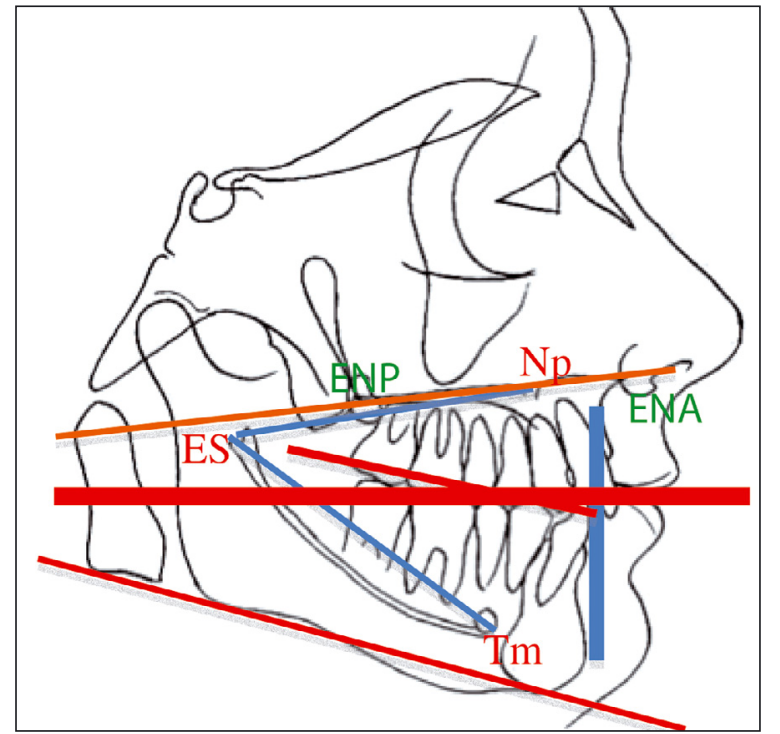

Figure 24

Analyse verticale de la position de molaires (d'après Danguy [12-14]).

Suite à une interposition linguale latérale [28,29], les molaires mandibulaires ne peuvent s'égresser, mais les molaires maxillaires, ayant eu un contact proprioceptif occlusal atypique, se sont égressées. Elles dépassent ainsi la ligne d'occlusion de Danguy dans la région molaire et prémolaire, avec une courbe d'occlusion particulièrement accentuée.

La ligne d'occlusion est tracée du rebord incisif mandibulaire (point situé sur la ligne APog à $2 \mathrm{~mm}$ au dessus de la bissectrice de l'angle Np-Es-Tm) à l'intersection des plans bispinal et mandibulaire de Downs (Fig. 24).

Si les molaires sont situées à distance du plan d'occlusion, il existe une infra-alvéolie molaire.

\subsubsection{Béance incisive}

Claire, 30 ans, angoissée, présente une béance de 5 à 5 après condylectomie suite à une hypercondylie droite (Fig. 25 et 26). La solution thérapeutique est ortho-chirurgicale avec chirurgie bimaxillaire d'avancée et abaissement maxillaire avec disjonction maxillaire et avancée mandibulaire suivie d'une rééducation linguale (Fig. 27 et 28).

Lanalyse architecturale met en évidence une infra-alvéolie incisive mandibulaire majeure (Fig. 29).

La chirurgie a replacé les procès alvéolaires et les incisives dans une situation idéale permettant la récupération d'un guide incisivo-canin fonctionnel,

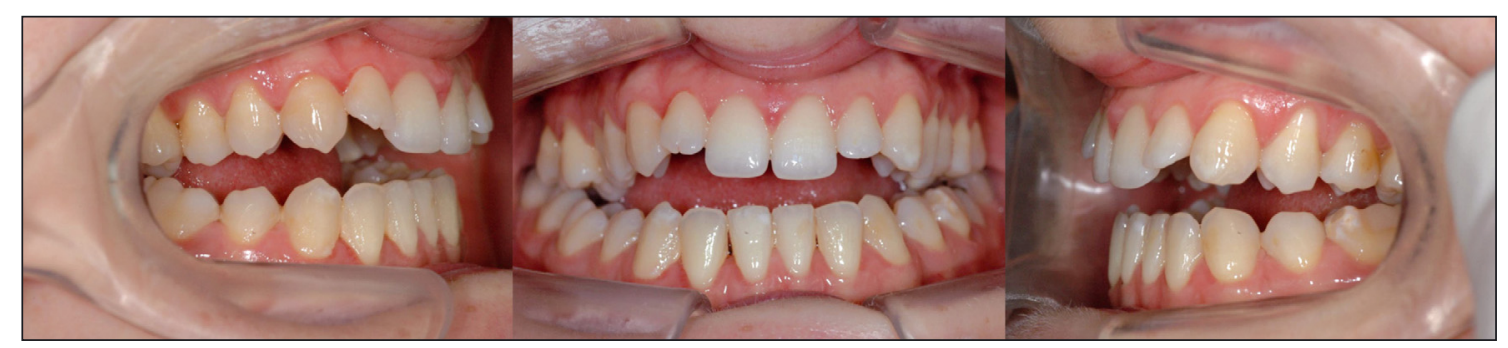

Figure 25

Cas de béance incisivo-molaire (C Bocquet).

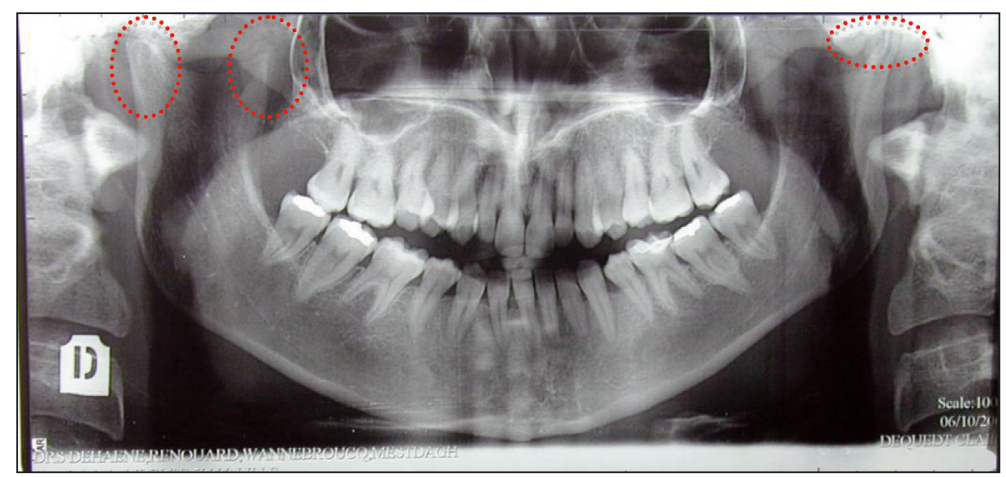

Figure 26

Cliché panoramique montrant le remaniement condylien (C) Bocquet). 

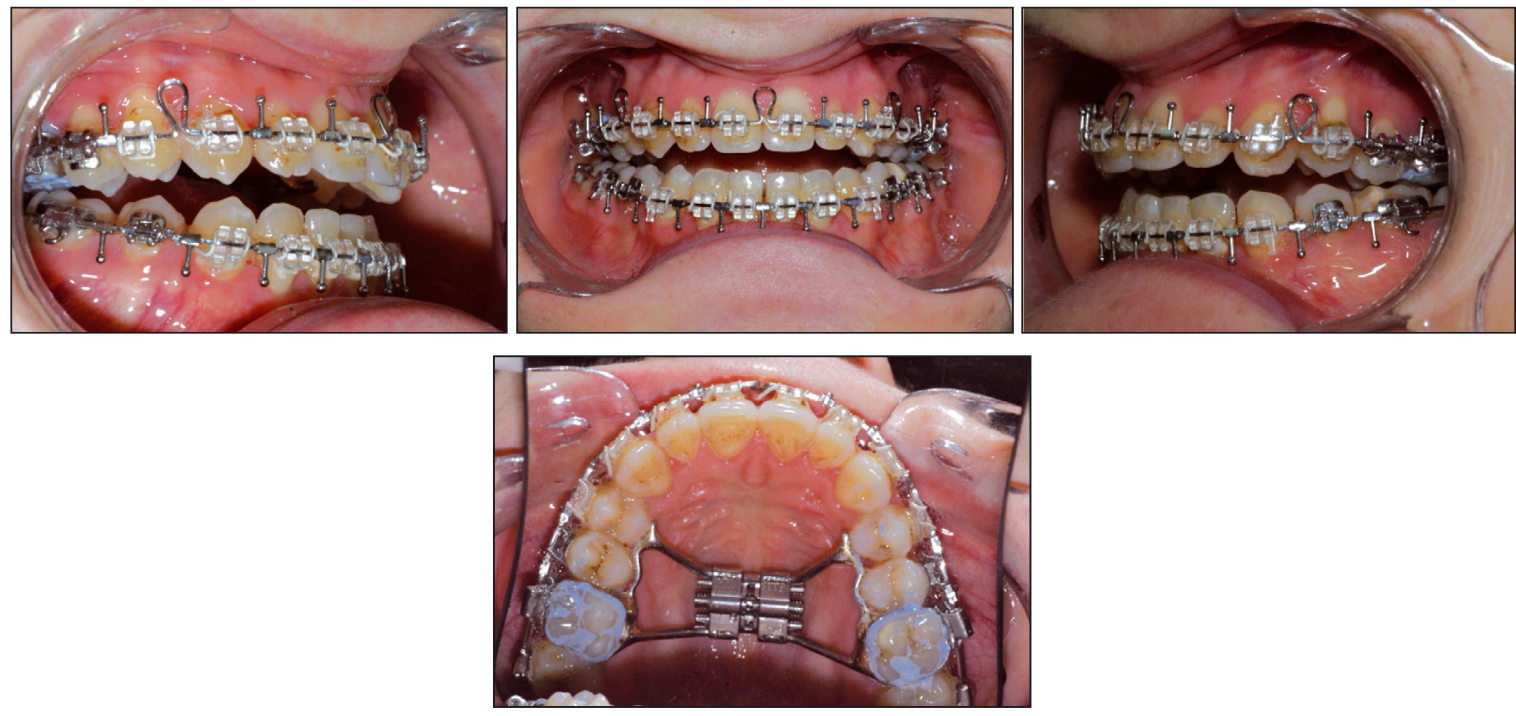

Figure 27

Vues endo-buccales avant chirurgie (c) Bocquet).
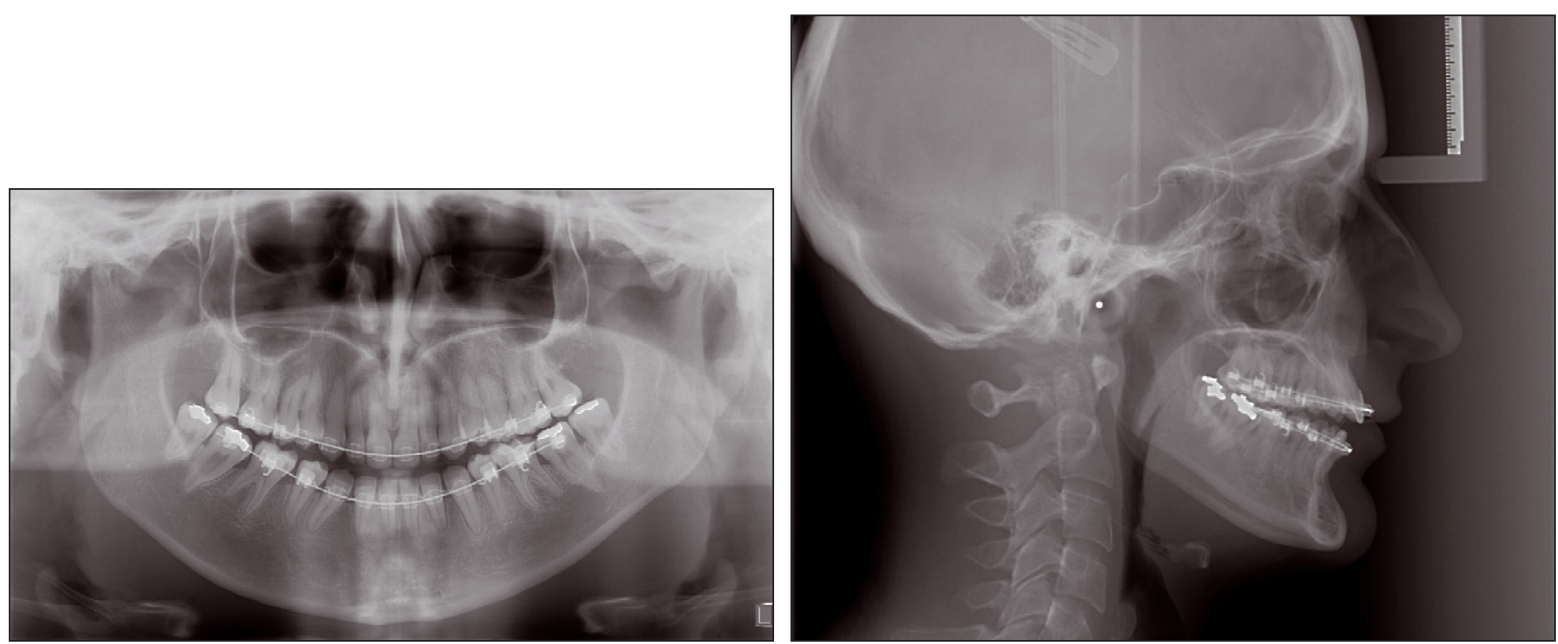

Figure 28

Clichés radiographiques avant chirurgie (C) Bocquet).

source de sécurité pour le maintien d'une fonction articulaire pérenne (Fig. 30, 31 et 32).

\subsubsection{Absence de liberté fonctionnelle}

Guillaume, 21 ans, consulte suite à des claquements articulaires qui se sont estompés et ont laissé place à des craquements avec douleurs.

L'examen clinique met en évidence une luxation irréductible de l'ATM droite sur un schéma occlusal de classe 2, division 2 (Fig. 33 et 34). Lavulsion des dents de sagesse sera à réaliser sous anesthésie locale pour éviter tout risque articulaire.
Le patient présente une rétro-alvéolie incisive maxillaire, une supra-alvéolie incisive mandibulaire, ceci entrainnant un blocage mandibulaire (Fig. 35).

La thérapeutique va être en premier lieu orthodontique pour vestibuloverser et égresser les incisives maxillaires, puis articulaire par gouttière de distension, suivie par la phase de stabilisation orthodontique permettant l'égression molaire (Fig. 36).

Après traitement articulaire par gouttière de distension $(4 \mathrm{~mm})$ et avant la stabilisation orthodontique, l'espace créé par la néodiscisation est bien visible. Le traitement orthodontique de stabilisation permettra d'égresser les molaires (Fig. 37). 


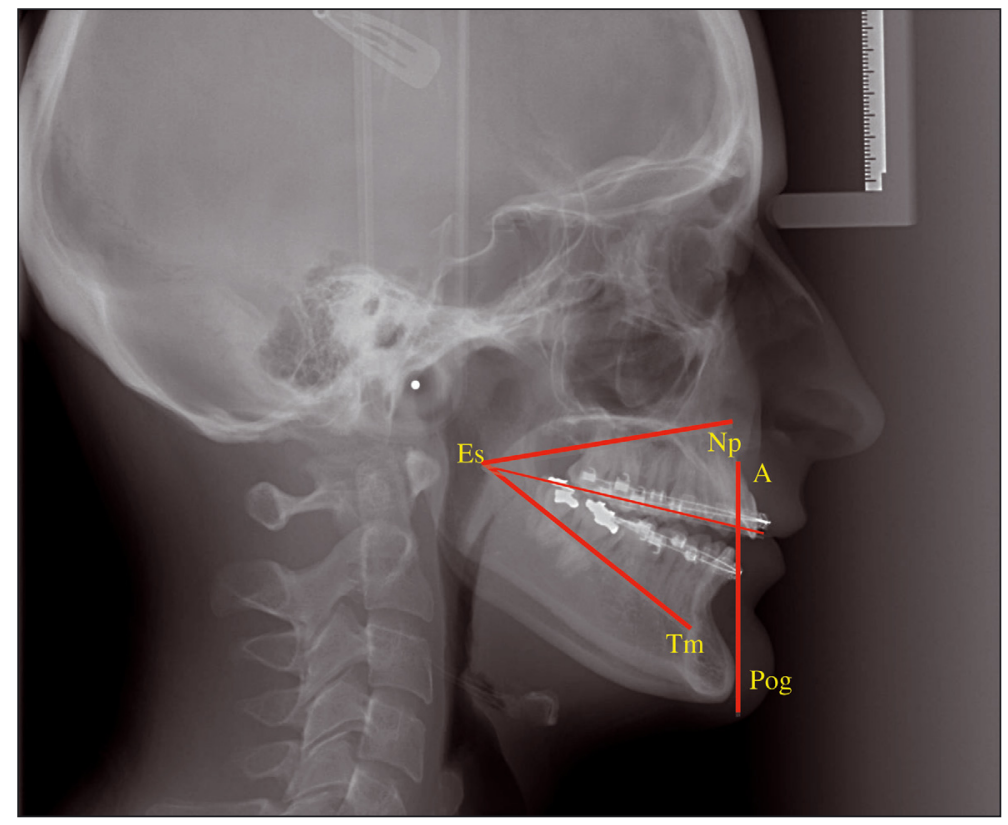

Figure 29

Analyse verticale de la position des incisives avant chirurgie (C) Bocquet).
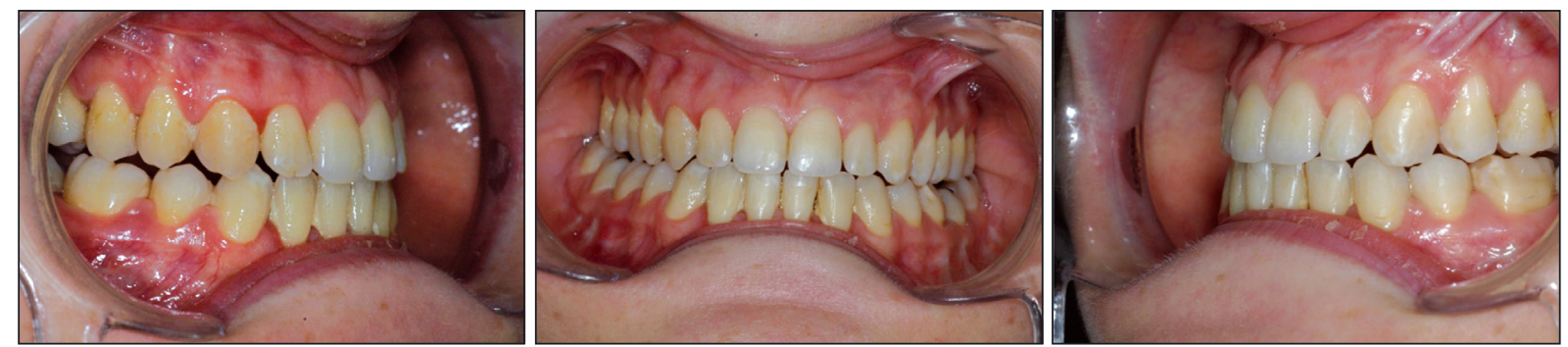

Figure 30

Vues endo-buccales après chirurgie (C Bocquet et Deffrennes).

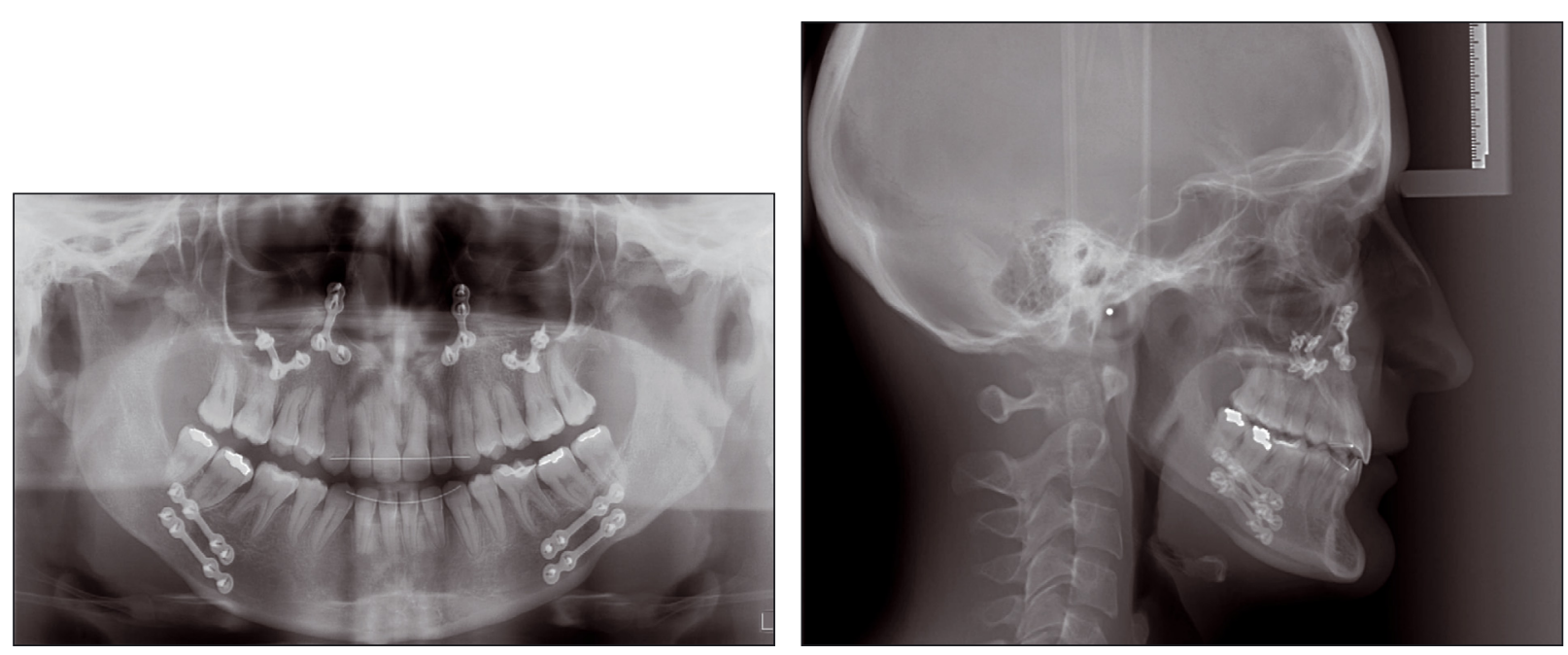

Figure 31

Clichés radiographiques après chirurgie (C) Bocquet et Deffrennes). 


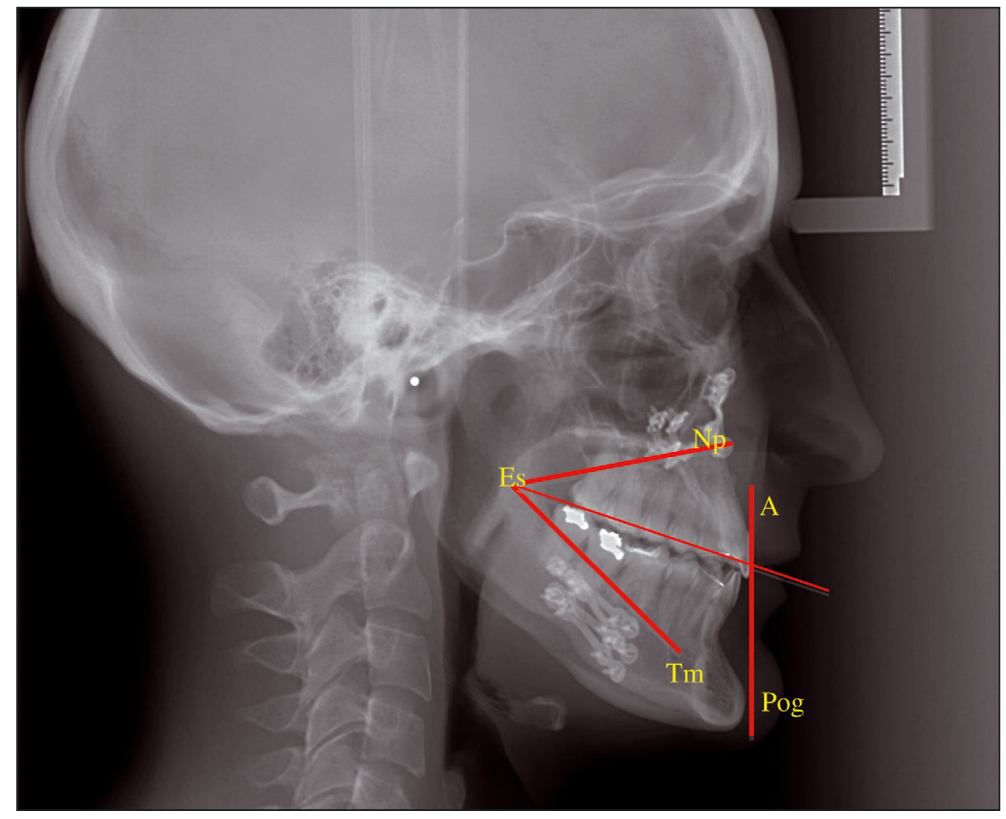

Figure 32

Analyse verticale des incisives après chirurgie (C) Bocquet).

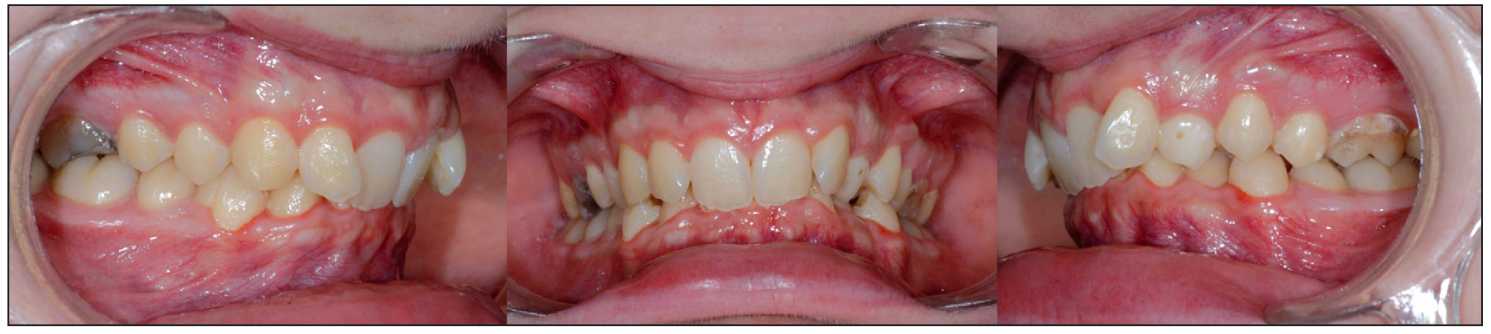

Figure 33

Vues endo-buccales (@ Bocquet).

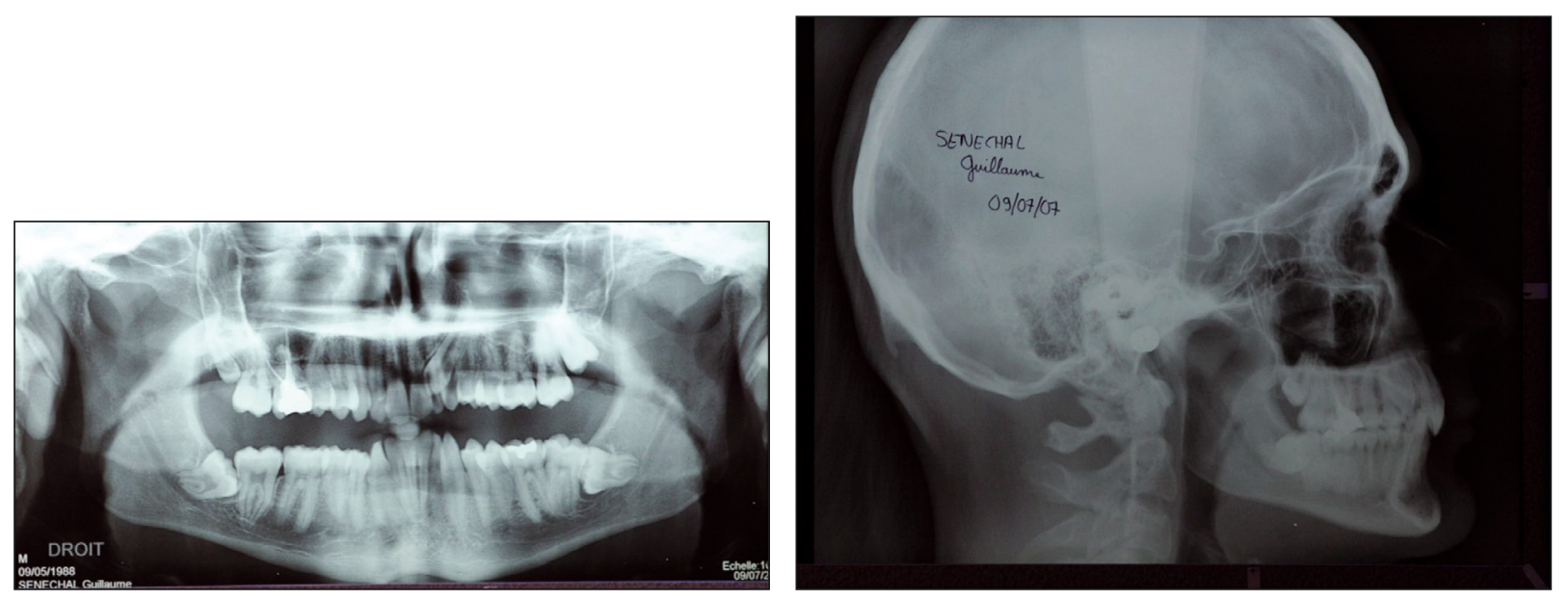

Figure 34

Clichés radiographiques (ㄷ Bocquet). 


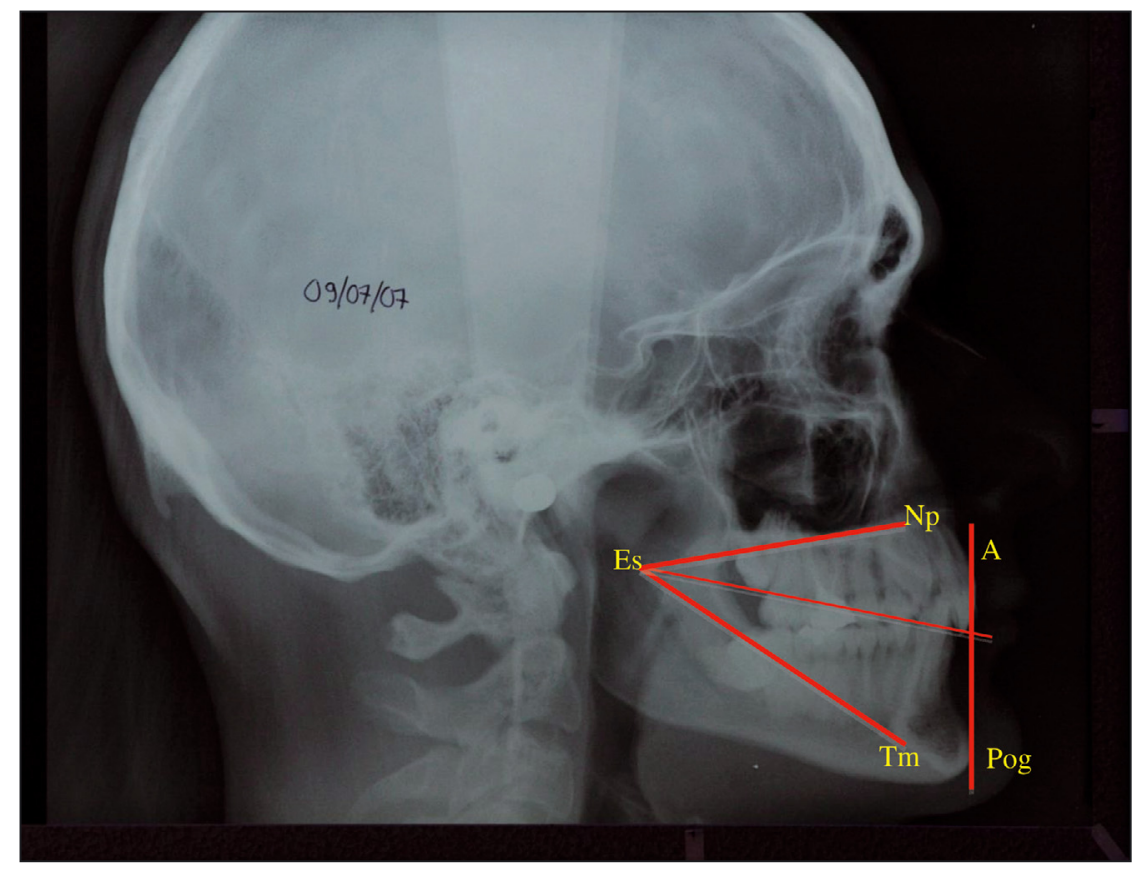

Figure 35

Analyse verticale des incisives (C) Bocquet).

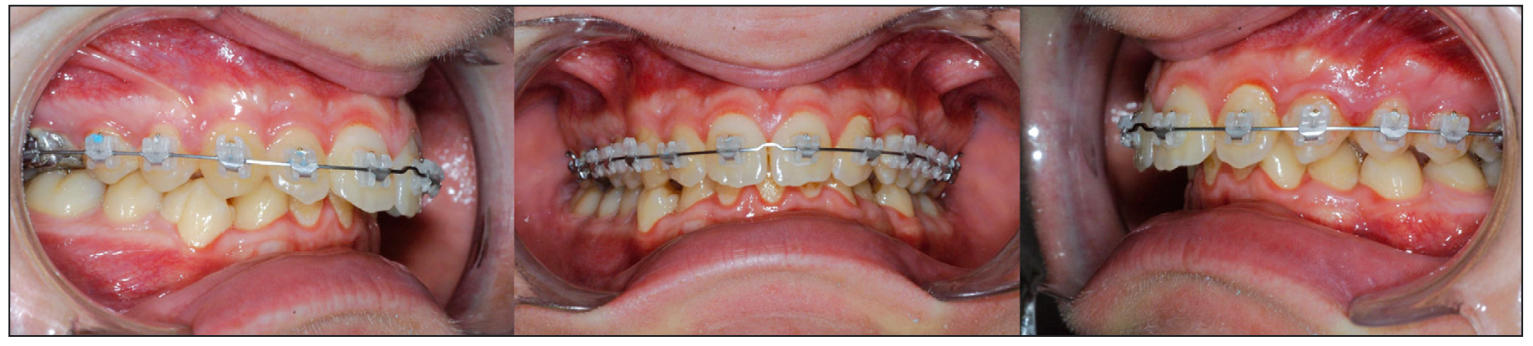

Figure 36

Vues endo-buccales après la première phase orthodontique (C Bocquet et Jarcet-Walkowiak).

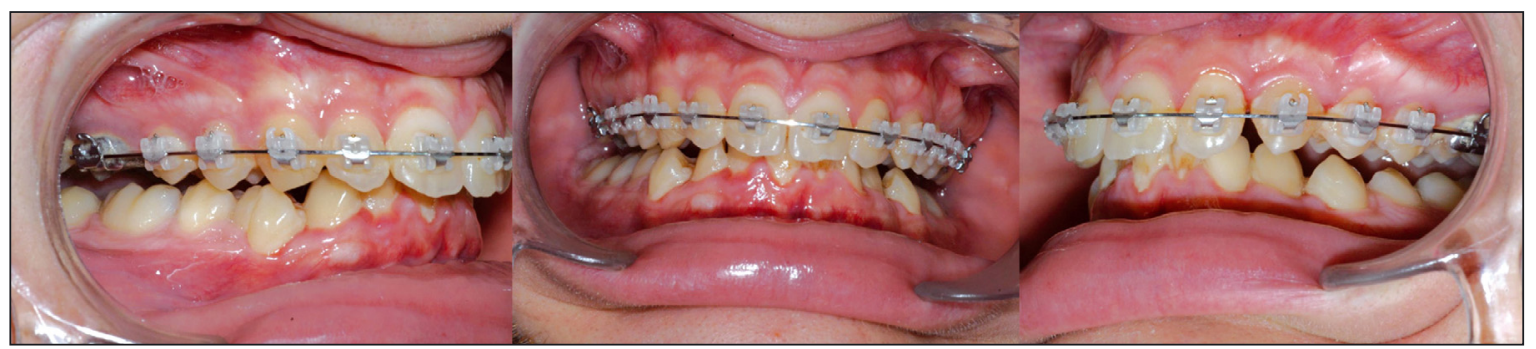

Figure 37

Vues endo-buccales après traitement articulaire (C) Bocquet).

\section{Conclusion}

Lanatomie, la physiologie, la cinétique, la pathologie de l'ATM et la thérapeutique articulaire sont des domaines complexes.

Lorthodontie est retenue comme un moyen thérapeutique dans le traitement d'une algie et/ou d'une dysfonction de l'appareil manducateur. L'orthodontie sera à manier avec rigueur après un examen clinique précis, des examens complémentaires radiologiques choisis, l'établissement d'un diagnostic étiopathogénique rigoureux afin d'appliquer la thérapeutique appropriée, thérapeutique qui pourra faire appel à d'autres disciplines puisque les étiologies des 
dysfonctions temporo-mandibulaires sont multifactorielles $[3,5,6]$.

De plus, l'orthodontiste devra respecter certaines règles :

- baguer les arcades entières,

- arrêter le port de tractions intermaxillaires en cas d'apparition de douleurs, claquements ou craquements : les tractions intermaxillaires (TIM) de classe II hyperpropulsent la tête condylienne et distendent le frein méniscal, les TIM de classe III entraînent une compression dans le fond de la cavité glénoïde,

- éviter toutes tractions intermaxillaires en cas de trouble temporo-mandibulaire préexistant,

- rétracter les incisives en respectant la pente condylienne (torque et forces douces) afin de permettre une propulsion mandibulaire naturelle,

- contrôler les interférences en fin de traitement,

- faire coïncider l'intercuspidation maximale et la relation centrée,

- contrôler les mouvements de latéralité,

- contrôler l'évolution des dents de sagesse et préférer leur avulsion sous anesthésie locale.

\section{Bibliographie}

[1] Autet BM. Examen ostéopathique prenant en compte l'activité tonique posturale. Mémoire de la Sereto. Montpellier, 1985.

[2] Bonnier L. La modification de l'occlusion a-t-elle une répercussion immédiate sur le système postural fin ? In: M. Lacour. Posture et environnement. Montpellier: Sauramps médical, 1997:77-86.

[3] Bonnier LR, Marucchi C. Système tonique postural et occlusion dentaire. En quoi ophtalmologistes et chirurgiensdentistes sont-ils concernés ? In: M. Lacour. Posture et équilibre. Montpellier: Sauramps médical, 1999:147-153.

[4] Boulaadas M, Boumediane H, El Gbouri H, Nassih M, Serghrouchni H, Zin R, et al. Exploration radiologique de l'articulation temporo-mandibulaire. Médecine du Maghreb 1997;61.

[5] Bourdiol H. Rôle de l'orthodontiste dans la prévention du dysfonctionnement cranio-mandibulaire. C.N.O. 6e journée internationale. Paris, 1989.

[6] Bricot B. Troubles statiques et SADAM. C.N.O. Ge journée internationale. Paris, 1989.

[7] Caix P. Larticulation temporo-mandibulaire et son vrai faux ménisque (approche morphologique, fonctionnelle, clinique et chirurgicale). Thèse Doct Etat Biol Hum. Amiens, 1991.

[8] Cardonnet M, Clauzade M. Diagnostic différentiel des dysfonctions de l'ATM. Cah Proth 1987;57:125-170.
[9] Cheynet F, Guyot L, Richard O, Layoun W, Gola R. Discomallear and malleomandibular ligaments: anatomical study and clinical applications. Surgical and radiology anatomy 2003;25:152-157.

[10] Clauzade M, Darraillans B. L'homme, le crâne, les dents. Perpignan: S.E.O.O., 1992

[11] Clauzade M, Marty JP. Orthoposturodontie. Perpignan: S.E.O.O., 1998

[12] Danguy M, Danguy C. Une analyse architecturale de la dimension verticale. Rev Orthod Fr 2002;73:365-376.

[13] Danguy M, Danguy-Derot C. Détermination de la situation verticale de l'incisive médiale maxillaire. A.J.O. Edition Française, Janvier 1998.

[14] Danguy M, Danguy-Derot C. A propos d'une analyse céphalométrique architecturale orthodontique. Rev Orthod Fr 2002;73:363-373.

[15] Deblock L, Vidailhet B. Les luxations de l'articulation temporo-mandibulaire: ATM et équilibre des fonctions oro-faciales. Rev Orthop Dento-Faciale 2000;34:155-173.

[16] Deblock L, Vidailhet B, Mahler P. Les luxations de l'articulation temporo-mandibulaire : étiopathogénie, une nouvelle hypothèse. Rev Orthop Dento-Faciale 2000;35:21-41.

[17] Decker A, Deffrennes D, Guillaumot G, Kohaut JC. Rôle de l'orthopédie dento-faciale dans la genèse, le traitement et la prévention des dysfonctions cranio-mandibulaires. Rev Orthop Dento-Faciale 1993;27:433-459.

[18] Deffrennes D, Negrier B, Hadjean E. Place de la chirurgie dans les dysfonctions temporo-mandibulaires. Orthod Fr 1990;61:797-811.

[19] Dupas PH. Nouvelle approche du dysfonctionnement cranio-mandibulaire : du diagnostic à la gouttière. Paris: CDP, 2005: 216 p.

[20] Durand B, Jeantet M, Delsol L, Nègre J. Les dysfonctions cranio-mandibulaires: quelle orientation thérapeutique? Chir Dent de Fr 1998;68:96-99.

[21] Fontaine C, Chevrel JP. Anatomie clinique. Volume 3: Tête et cou. Paris: Springer-Verlag, 1996.

[22] Fricain JC, Marteau JM. Diagnostic des dysfonctions crânio-mandibulaires dynamiques dans le sens antéropostérieur : intérêt de l'imagerie par résonance magnétique. MBCB 1997;3(2):75-82

[23] Gagey PM, Weber B. Posturologie. Régulation et dérèglements de la station debout. Paris: Masson, 2e ed, 1999.

[24] Garcia R, Deffrennes D, Simon A. Les luxations méniscales irréductibles de l'articulation temporo-mandibulaire. Approche diagnostique et thérapeutique. Rev Orthop DentoFaciale 1994;28:151-180.

[25] Gola R, Cheynet F, Richard D, Guyot L. Faut-il opérer les syndromes algo-dysfonctionnels de l'appareil manducateur (SADAM) ? e-mémoires de l'Académie Nationale de Chirurgie 2005;4(3):30-41.

[26] Gola R, Chossegros C, Cheynet F. Les ligaments oto-mandibulaires : ligaments disco-malléaire et malléo-mandibulaire. Rev Stomatol Chir Maxillofac 1997;98(2);66-71.

[27] Hüe O. Manuel d'occlusodontie. Paris: Masson, 1992. 
[28] Jaïs L. Approche clinique et thérapeutique du rôle de langue dans certaines asymétries posturales. In: M. Lacour. Posture et environnement. Montpellier: Sauramps médical, 1997:103-108

[29] Jaïs L, Weber W. La position de la langue modifie-t-elle certains critères stabilométriques? In: M. Lacour. Posture et équilibre. Montpellier: Sauramps médical, 1999:155-164.

[30] Le Gall MG, Lauret JF. Occlusion et fonction. Une approche clinique rationnelle. Paris: CDP, 2002.

[31] Korn M, Massol JP, Serfaty E. L'orthodontie posturale. Rev Orthop Dento-Faciale 1995;29:301-317.
[32] Mongini F, Schmid W. Orthopédie cranio-mandibulaire et articulaire (A.T.M). Paris: CDP, 1998.

[33] Monzani D, Setti G, Chiesi C, Galetti R. Désordres craniomandibulaires et phorie : une approche multidisciplinaire. In: M. Lacour. Posture et équilibre. Montpellier: Sauramps médical, 1999:165-177.

[34] Roth RH. Temporomandibular pain-Dysfunction and occlusal relationships. Angle Orthod 1976;43:136-153.

[35] Slavicek R. Les principes de l'occlusion. Rev Orthop Dento Faciale 1983;17:449-490.

[36] Unger F. Pratique clinique des orthèses mandibulaires. Paris: CDP, 2003. 